\title{
Optimal Design of Limited Partnership
}

\section{Agreements}

\author{
Mohammad Abbas Rezaei*
}

April 16, 2021

\begin{abstract}
General partners (GP) are sometimes paid on a deal-by-deal basis and other times on a whole-portfolio basis. When is one method of payment better than the other? I show that when assets (projects or firms) are highly correlated or when GPs have low reputation, whole-portfolio contracting is superior to deal-by-deal contracting. In this case, by bundling payouts together, whole- portfolio contracting enhances incentives for GPs to exert effort. Therefore, it is better suited to alleviate the moral hazard problem which is stronger than the adverse selection problem in the case of high correlation of assets or low reputation of GPs. In contrast, for low correlation of assets or high reputation of GPs, information asymmetry concerns dominate and deal-bydeal contracts become optimal, as they can efficiently weed out bad projects one by one. These results shed light on recent empirical findings on the relationship between investors and venture capitalists.
\end{abstract}

Key Words: Limited Partnership Agreement, Compensation Timing, Portfolio Choice.

\footnotetext{
*Haas School of Business at UC Berkeley. Email address: mohammad_rezaei@haas.berkeley.edu
} 


\section{Introduction}

In private equity, the relationship between fund managers (general partners or GPs) and investors (limited partners or LPs) is governed by a "limited partnership agreement" (LPA). These contracts are crucial in determining how GPs behave for the following reasons. First, LPs have limited resources outside of these contracts to discipline GPs. Second, these agreements typically remain in effect for about a decade, and recently up to 15 years, (with little room for renegotiation). Finally, GPs' actions are hard to observe and writing a contract which provides the right incentives for GPs is of critical importance.

In general, there are three main financial components in an LPA. These are the management fee, carried interest, and the method of payments to GPs. While the structure of the management fee and carried interest has been the subject of extensive research, there is virtually no theory on why the method of payment is important and how it effects GPs' performance. Historically LPAs offer two methods for paying carried interest to GPs. The first method is deal-by-deal or "American". This provision allows GPs to earn the interest as soon as each deal is exited. The second method is whole-fund or "European". In this method, LPs receive the entire interest on their investment(s) before GPs get any carried interest 1

At first glance, it seems that the European method is more favorable to LPs--in fact, Huther et al (2020) calls it the "LP-friendly contract". In particular, if we assume that the GP does not change her strategy under different types of contracting, then whole-portfolio contracting is preferred to the deal-by-deal method for investors. However, as the GP changes her strategy as the contract changes, it is not clear which method is more efficient for investors. Here is an example which illuminates the difference between these two methods. Suppose a GP has invested in a fund consisting of two firms. Suppose one of the firms exits with a high return but the other one loses money so that in total the return is low. In a dealby-deal contract, the GP would get some interest on the successful exit. However in the whole-portfolio method, since the low-return investment offsets the high-return one, the GP will receive almost nothing and the whole return will go to the investor. 2

To fix ideas, consider the following scenario. Suppose there is an LP who wants to invest in a pool of two projects but has no expertise to find profitable investment opportunities. As

\footnotetext{
${ }^{1}$ In Litvak (2009), there is detailed explanation on different provisions for these methods.

${ }^{2}$ Even in the presence of claw-back provisions which requires GPs to return some of the return at the end to the LP, still the GP gets an interest free loan from the LP in the meantime. Moreover in the sample of contracts considered in Cumming and Johan (2009), only about $26 \%$ of contracts had claw-back.
} 
a result, he hires a GP to do the job. The GP has to exert effort to find good investment opportunities, but even with significant effort she may end up with low quality projects. As is prevalent in this setting, the LP has no control over the GP's actions, nor does he know the quality of the projects, unlike the GP. Thus, the contracting is subject to both moral hazard and adverse selection.

Within the setting outlined above, I investigate the conditions under which each method of payment (deal-by-deal or whole-portfolio) is optimal. As a result, I can explain some empirical findings documented in the literature. First, I show that when projects are highly correlated, whole-portfolio contracting is optimal for the LP. As the correlation declines, the space of portfolios where deal-by-deal contracting is preferred expands. This phenomena has been documented empirically in Magro (2018). The mechanism behind this result comes from the trade-off between the moral hazard about the effort to find good projects versus the information asymmetry about the quality of projects. When projects have high correlation, bundling the performance of projects together can enhance incentives for the GP to exert effort on them. In this case, even when projects are subject to different degrees of adverse selection, the loss of efficiency is still low enough that whole-portfolio contracting is preferred to deal-by-deal contracting which can handle adverse selection efficiently.

Second I show that when the GP is not reputable, it is more likely that the LP should use whole-portfolio contracting compared to when contracting with a reputable agent. 3 This result is in alignment with the findings in Huther et al (2020). In this paper, the authors propose that when a GP is more reputable, they have more market power and hence can get more favorable contracting terms. In my setting, however, this comes from the fact that for non-reputable agents, whole-portfolio contracting can reduce the chance of making bad investments, hence improving the investment strategy. Therefore, the sorting effect exists in this environment, but indirectly as a result of the change of behavior of the agent due to the terms of the contract.

The model yields other results and predictions. For example, I show that when there is little or no information asymmetry about the quality of projects between investor and agent, whole-portfolio contracting is the dominant form of contracting. This can explain why we see this form of contracting when the underlying assets are public firms. Specifically in the case of hedge funds or mutual funds, the payout to the agent is almost always a function of

\footnotetext{
${ }^{3}$ By non-reputable agent, I mean an agent that investor can not verify her access to investment opportunities hence needs to be distinguished from fly-by-night operators.
} 
the performance of the whole portfolio rather than the individual performance of assets in the portfolio. I also predict that investors' information can affect the method of payment. When investors are not fully informed on the structure of an investment, they prefer to have a narrower scope of investment (hence higher correlation) and use the whole-portfolio contracting method.

The main feature of the model which enables me to show these results is the fact that projects are heterogeneous. If different projects are always subject to same degree of moral hazard and information asymmetry, then bundling the payouts together has no efficiency loss and whole-portfolio contracting is the dominant method of contracting, as is the case for many contracts in the real world. This is the dominant assumption in the literature, in the seminal work of Diamond (1984) and subsequent studies. For example, Laux (2001) considers a pool of homogeneous projects and show how investors can design better contracts by the pooling and loosening of limited liability. However, when a typical VC invests in a pool of projects, it is reasonable to assume a high degree of heterogeneity between projects 4

The heterogeneity of projects creates a trade off between moral hazard and adverse selection. When a contract is written on the whole-portfolio basis, investors can more easily persuade agents to exert effort on the projects through bundling the payouts. However, whole-portfolio contracting takes away the flexibility to deal with the different degrees of adverse selection that the projects are subject to. For higher correlation between projects or lower reputation of agents, the priority is to mitigate the more severe moral hazard problem, and the whole-portfolio contracting is therefore preferred. On the contrary, when the correlation between projects is low or the agent is reputable, adverse selection is more severe, and deal-by-deal contracting is better suited to deal with this issue.

This paper relates to the theoretical literature in the area of PE funding. In Axelson et al (2009), the authors study the problem of leverage in buyouts and show that a combination of ex-ante pooled financing and ex-post deal-by-deal financing is optimal. In their setting, the timing of the investment on projects is different, while in a lot of limited partnership contracts the GP is required to choose the portfolio firms early in the life-span of the LPA. In another similar work, Fang (2019) shows why LPs restrict the investment timing of GPs. In both of these works, the authors abstract away from the moral hazard problem

\footnotetext{
${ }^{4}$ VCs invest in projects which are highly innovative with unique business plans with very few assets in place unlike for example banks which give loan to ordinary businesses or mortgages to residential/commercial properties. As a result, we expect much more heterogeneity in VCs invested portfolios.
} 
between LPs and GPs, and also consider a pool of similar projects. Because of the homogeneity between projects, when the method of financing is restricted to ex-ante, whole-portfolio financing is always optimal in their setting and they are not able to explain the abundance of the deal-by-deal ex-ante contracting in the PE industry.

This work also contributes to the literature on investment pooling and portfolio contracting. Inderst et al (2007) consider the case in which investors faces multiple agents and investment pooling and credit rationing can motivate optimal investment strategy. Their main mechanism relies on the competition among agents, while in my work credit rationing has no bite as investors face only one agent. Fulghieri and Sevilir (2009) also consider the case of contracting between an investor and multiple agents and focus on the double moral hazard problem between GPs and entrepreneurs. In contrast, I abstract away from GP/entrepreneur problems and focus on the contracting between GPs and LPs. This paper also relates to the literature on moral hazard with learning, He et al (2017) and Miao and Rivera (2016), and experimentation and Bandit problems, Pourbabaee (2020), as well.

Empirically, the first work which addresses the importance of the method of compensation in VC settings is Litvak (2009). She shows that the shift in the timing of compensation can affect the present value of the payment to the GP as much as changing the contracting terms themselves. While the importance of the compensation method is discussed in Litvak (2009), Huther et al (2020) and Magro (2018) study the effects of payment methods on the GP investment strategy and fund's return. All of these papers are empirical and offer little theory on the matter.

More broadly, the first work which studies GP compensation is Gompers and Lerner (1999). The authors explore the cross sectional and time variation of the management fee and carried interest in the contract terms, assuming that contracts have the same method of payment. Metrick and Yasuda (2010) study a similar problem using an option-pricing framework, and focus more on buyout funds. Unlike these works, Robinson and Sensoy (2013) have access to cash flow data as well as contracting terms, which links the management payment to the performance using a novel data set containing all the payment from a big institutional investor to GPs.

The paper proceeds as follows. Section 2, introduces the models and shows the optimal contracting on one project. In Section [3, I solves the problem of optimal whole-portfolio contracting and compare it to the deal-by-deal contract. Section 4 consider the same prob- 
lem for non-reputable GPs and I compare the results to the case of reputable agents. Section 5 considers various extensions of the model. Finally Section 6 concludes.

\section{Model}

There are three classes of agents in the model: limited partners (investors or LPs), general partners (GPs) and fly-by-night operators (FNOs). All agents are risk-neutral and have access to a safe asset technology with a return which is normalized to zero. There are two types of general partners, reputable and non-reputable. Both types of general partners have access to a pool of projects in which they can invest in. The limited partner has capital which is needed to run projects 5 FNOs have no access to the pool of risky projects but they can mimic the behavior of a GP. I assume that if the GP is reputable, then the LP can verify that she has access to projects. However, the LP can not distinguish between a nonreputable agent and a FNO. Initially, I focus on reputable GPs and discuss contracting with non-reputable GPs in Section 4. Every project needs an investment outlay of $I$. The GP has no initial money and should raise it from the LP if she decides to invest in the project(s) 6 There are two types of projects, $\theta \in\{G, B\}$. A good project (type $G$ ) has guaranteed return $R$ (hence it is always successful) but a bad project (type $B$ ) has return $R$ with probability $p$ and return 0 with probability $1-p$. The GP can also opt to not invest in a project and invest the raised capital in a safe asset, therefore receiving the return $I$. Hence, the possible outcomes are $\{0, I, R, 2 I, R+I, 2 R\}$ if the GP raises enough capital for two projects $(2 I)$. Clearly, if the GP raises only $I$, then possible returns are $\{0, I, R\}$. Type $B$ projects are negative NPV, so I assume

\section{Assumption 1}

$$
p R<I
$$

The GP can exert effort to increase the chance of getting a good project. Moreover, if the GP exerts no effort for a project, then the project which is chosen is guaranteed to be bad (type $B$ ). Otherwise, if the GP exerts a binary effort with cost $c$, the chance of getting a good project (type $G$ ) is $\lambda$. I assume that the decision to exert effort is optimal in the following sense

\section{Assumption 2}

$$
\lambda R+(1-\lambda) I>I+c
$$

\footnotetext{
${ }^{5}$ Throughout the paper, I use he/him to refer to the LP and she/her to refer to the GP.

${ }^{6}$ The assumption that GP has no initial capitl has no effect on the results. We can assume that GP needs extra capital $I$ as long the payout of the contract to the GP is at least as her initial capital.
} 
which can be written as

$$
R-\frac{c}{\lambda}>I
$$

However, it is possible that the agent exerts effort but does not commit to not invest if the quality is bad. In this case, the return is

$$
\lambda R+(1-\lambda) p R
$$

which is less than $\lambda R+(1-\lambda) I$ by assumption 1, It can be seen that if there is no agency friction, then when equation (1) holds, the agent/investor exerts effort to obtain a good project and invest in the project if he ends up with a type $G$, otherwise keeping the money in a safe asset. In this case, the profit made from the project is $\lambda(R-I)-c$. In my definition $\frac{1}{\lambda}$ measures the extent of the moral hazard issue. Higher $\lambda$ means higher chance of obtaining a good project, so the moral hazard problem is less severe. On the other hand, $p$ measures the extent of the information asymmetry between agents, since for higher $p$ it is harder to give incentives for the GP to not invest in a bad project.

The model has three dates $t=0,1,2$ and two periods. At $t=0$, the contract between the GP and the LP is written and capital is raised. Then between dates 0 and 1 , the GP can exert effort to increase the chance of getting a good project. At $t=1$, the type of projects is revealed to the GP and she makes the investment either in these projects or safe assets. Finally, at $t=2$, cash flows are realized and agents receive their money based on the contract. In the real world, it is possible that projects exit at different times but if the contract is based on whole-portfolio performance, then money is stored in an escrow account until distributed later when all the projects exit. Hence my assumption on having the same exit time is not unrealistic.

\subsection{Deal-by-deal contract}

In this section, I consider the contracting problem when the contract between the GP and the LP is written in a deal-by-deal way. Since agents are risk-neutral, the optimal deal-bydeal contract consists of two optimal contracts on a single project. Therefore, I only need to study the contracting problem for one project.

In order to fund projects, claims $s_{G P}(x)=s(x)$ and $s_{L P}(x)=x-s_{G P}(x)$ are issued, which determines how much agents will receive when the payout of the project is $x$. I impose following a priori assumptions on the payout of securities.

- Limited Liability: $0 \leq s_{G P}(x), s_{L P}(x)$. 
- Monotonicity: $s(x)$ and $x-s(x)$ are non-decreasing in $x$ when $x$ is an outcome on the equilibrium path.

This monotonicity assumption is common in the literature on security design-See Nachman and Noe (1994), for example. Sometimes the security is assumed to be monotonic on the whole possible set of payouts. . I will revisit this issue in Section 8 ,

The LP can not observe the quality of the chosen project (projects) or if the GP exerts effort or not. However, the LP can observe whether the GP invests in the project or in the safe asset. Also, the cash flow is verifiable at the end of period 2 as well.

For one project, after the issuance of $s(x)$, there are four possible strategies by the GP.

1. Do not invest: The return to GP is $s(I)$.

2. Invest with no effort: The return is $p s(R)$.

3. Exert effort and invest regardless of quality: $\lambda s(R)+(1-\lambda) p s(R)-c$.

4. Exert effort and invest only in the good project: $\lambda s(R)+(1-\lambda) s(I)-c$.

Clearly, the optimal strategy is the fourth one if there was no agency friction (I will show later that strategy (4) is also optimal even in the presence of agency friction.) . Assuming this, here is how the LP can implement strategy (4). The scheduled payment's system $(s(I), s(R))$ induce the GP to choose strategy (4) if and only if it satisfies

$$
\begin{aligned}
& s(R) \geq s(I)+\frac{c}{\lambda} \\
& s(I) \geq p s(R) .
\end{aligned}
$$

The first condition insures that the agent exerts effort to obtain a good project and the second one insures that the agent does not invest if the quality of the project turns out to be bad. As usual, we have the participation constraint by the LP which is

$$
E\left[s_{L P}(x)\right]=E[x-s(x)] \geq I .
$$

The problem faced by the LP can then be written as

$$
\max _{s(I), s(R)} E[x-s(x)]
$$

where $(s(I), s(R))$ satisfy equations (2) and (3). Inserting equation (3) in equation (2) gives

$$
s(R) \geq \underset{7}{p s(R)}+\frac{c}{\lambda}
$$




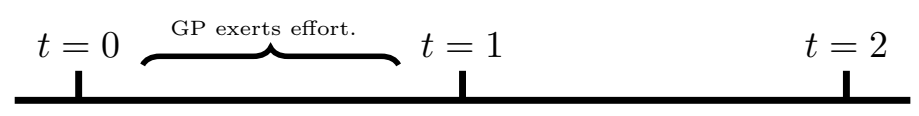
- Securities $s_{G P}$ and $s_{L P}^{\bullet}$ GP realises the type of Cash flows are realized.
are issued.

are issued.

- It is distributed

- $2 I$ is raised.

- Investment decision is according

made. $\quad$ to securities.

Figure 1: Timeline

hence $s(R) \geq \frac{c}{\lambda(1-p)}$. As a result, the optimal contract will be $\left(\frac{c}{\lambda(1-p)}, \frac{p c}{(1-p) \lambda}\right)$. Using equation (4), The project is funded if and only if we have

$$
\lambda\left[R-\frac{c}{\lambda(1-p)}\right]+(1-\lambda)\left[I-\frac{p c}{(1-p) \lambda}\right] \geq I
$$

Under this contract, the profit made by the GP is

$$
\begin{aligned}
& \Pi_{G P}=\Pi=\lambda \frac{c}{\lambda(1-p)}+(1-\lambda) \frac{p c}{\lambda(1-p)}-c \\
& =\frac{p c}{\lambda(1-p)}
\end{aligned}
$$

Since the optimal effort/investment strategy is chosen by the GP, we have $\Pi_{L P}+\Pi_{G P}=$ $\lambda(R-I)-c$. So the profit made by the LP from the contract on one project is

$$
\Pi_{L P}=\lambda(R-I)-c-\frac{p c}{\lambda(1-p)}
$$

We can see that for higher $p$, the LP makes less profit (and therefore the GP makes more). This is because, as mentioned before, higher $p$ is associated with more severe adverse selection and it makes it harder to motivate the GP to invest optimally since the outside option (the bad project) is more appealing. On the contrary, when $\lambda$ goes up, the profit goes up for the LP (and down for the GP) because the chance of success when exerting effort is higher, so less payment is needed to motivate effort. It is also worth noting that when there is no bad option for investment by the GP, which means $p=0$, then the LP can get the whole surplus of the project. This case is effectively means that there is no asymmetric information between the LP and the GP. As a result, in a setting with binary effort, contracting alleviates all the friction in the model. In section 5.1, I consider implications when there is more variance for effort in this special important case.

In order to compare the outcome of the results of different strategies induced by the LP, 
first note that the LP never induces strategy (1) as he personally has access to the safe asset. The second strategy has always negative NPV since the profit by the LP is

$$
\begin{aligned}
& p(R-s(R))-I \\
& \leq p R-I<0
\end{aligned}
$$

by assumption 1, Finally to optimally induce strategy (3), note that in this case the LP optimally sets $s(I)=0$ as $I$ is not the outcome of the induced strategy. To induce effort, the payout should satisfy

$$
s(R) \geq p s(R)+\frac{c}{\lambda}
$$

which implies $s(R) \geq \frac{c}{\lambda(1-p)}$. Hence the LP issues security $\left(0, \frac{c}{\lambda(1-p)}\right)$. The profit made by the LP by this contract is

$$
(\lambda+(1-\lambda) p)\left(R-\frac{c}{\lambda(1-p)}\right)-I
$$

This is less than the profit made by the LP by strategy (4) (equation (17)) by assumption 1 .

In summary, we have the following for investment on one project.

Proposition 1 (optimal deal-by-deal contract) The optimal strategy that the LP induces the GP to choose is strategy (4). Moreover, the security $(s(I), s(R))=\left(\frac{c}{\lambda(1-p)}, \frac{p c}{(1-p) \lambda}\right)$ is issued optimally by the LP to fund the project. The funding is possible if and only if

$$
\Pi_{L P}=\lambda(R-I)-c-\frac{p c}{\lambda(1-p)} \geq 0
$$

When there are two projects with parameters $\left(\lambda_{1}, p_{1}\right)$ and $\left(\lambda_{2}, p_{2}\right)$, in a deal-by-deal contract, the optimal contract for one project is written for each of the projects. Therefore the expected profit by the GP will be

$$
\Pi_{G P}=\sum_{i=1}^{2} \frac{p_{i} c}{\lambda_{i}\left(1-p_{i}\right)} .
$$

In the next section, we see how tying the payouts of the projects together can change the expected payout to the LP (and the GP). 


\section{Optimal Portfolio Contracting}

In this section, I analyze the question of optimal contracting when a portfolio of projects is chosen by the GP and the payout can depend on the whole return of the portfolio. Then I compare whole-portfolio contracting with deal-by-deal contracting to see how the investment environment can affect the choice of contract by investors. But first I need to introduce the dependency between projects, which I do in the next part.

\subsection{Correlation structure of the portfolio}

When the GP forms a portfolio of investments, not only the return and quality of each project is important, but the correlation structure between projects is important as well. In Magro (2018), the correlation structure in the invested portfolio under different types of contracting is studied, analyzing the correlation between projects in two dimensions of industry and geography.

Here I assume that the correlation between projects is given by a parameter $0 \leq \rho \leq 1$ which means that if the GP exerts effort on both projects, then

$\mathbb{P}[$ project 1 and 2 are good $\|$ effort exerted on both projects $]=\rho \min \left(\lambda_{1}, \lambda_{2}\right)$.

Here I assume that investors can observe $\rho$ and potentially write a contract conditioned on it. I relax this condition in section 5.2 . The cost of exerting effort on both projects is twice that of one project, $2 c$. The correlation structure is irrelevant when the contract is written on a deal-by-deal basis. This is because, by risk neutrality, deal-by-deal contracting is equivalent to writing a contract with two different agents. Therefore I only need to study the problem when the payout depends on the payout of both projects.

Before going into detail on the whole-portfolio contracting, let me introduce some preliminary results which are needed later in the discussion. As in the deal-by-deal case, the GP should not invest in the type $B$ project. Hence possible optimal outcomes from the projects are $2 I, R+I$ and $2 R$. These correspond to cases in which the GP comes up with zero, one or two good projects respectively. As a result, $I$ and $R$ are not possible outcomes if the GP makes optimal investment decisions. Hence to minimize the incentive for these outcomes, it is easy to show that the optimal contract satisfies

$$
s(0)=s(I)=s(R)=0 .
$$




\subsection{Whole-Portfolio Contract}

In this section, I want to see how the optimal contract should be written when the return is a function of the total payouts of the projects. This resembles whole-portfolio contracting. I then compare it to deal-by-deal contracting to find under which parameters each type of contracting is efficient .

When writing the contract on the whole portfolio, as we saw in equation (10), we have $s(I)=s(R)=s(0)$. Set

$$
(x, y, z)=(s(2 R), s(R+I), s(2 I))
$$

The LP needs to impose some restrictions on the payment to the GP to make sure that the GP only invests in good projects. These conditions are

$$
\begin{aligned}
& z \geq \max \left\{p_{1} y, p_{2} y, p_{1} p_{2} x\right\} \\
& y \geq \max \left\{z, p_{1} x, p_{2} x\right\} \\
& x \geq \max \{z, y\} .
\end{aligned}
$$

These inequalities make sure that the GP will invest in good projects and only in good projects (hence withholding money from bad projects). For example, when the agent comes up with two bad projects, the payout for not investing in any bad project $(z)$ is not less than the (expected) payout if the agent invests in one bad project ( $p_{i} y$ for $\left.i=1,2\right)$ or invests in two bad projects $\left(p_{1} p_{2} x\right)$. A similar explanation applies to $y_{i} \geq p_{3-i} x$ for $i=1,2$. These conditions therefore discourage the GP from making bad investment decisions. Also since $x \geq y \geq z$, the GP will invest in good projects when they are available rather than investing in the safe asset. Note that when the contract satisfies these conditions, the payout to the GP is increasing on the equilibrium as the GP does not invest in a bad project, hence satisfying the monotonicity condition. Set $\lambda_{\max }=\max \left[\lambda_{1}, \lambda_{2}\right]$ and $\lambda_{\min }=\min \left[\lambda_{1}, \lambda_{2}\right]$. In addition, the GP should have incentive to exert effort on both projects. This gives

$$
\begin{aligned}
& \rho \lambda_{\min } x+\left[\lambda_{1}-2 \rho \lambda_{\min }+\lambda_{2}\right] y+\left[1-\lambda_{1}-\lambda_{2}+\rho \lambda_{\text {min }}\right] z \\
& \geq \max \left\{z+2 c, \lambda_{\max } y+\left(1-\lambda_{\max }\right) z+c\right\}
\end{aligned}
$$

The LHS term is the expected payout to the GP if she exerts effort on both projects. On the RHS we have expected payouts if no effort is exerted or if it is exerted on only one project. 


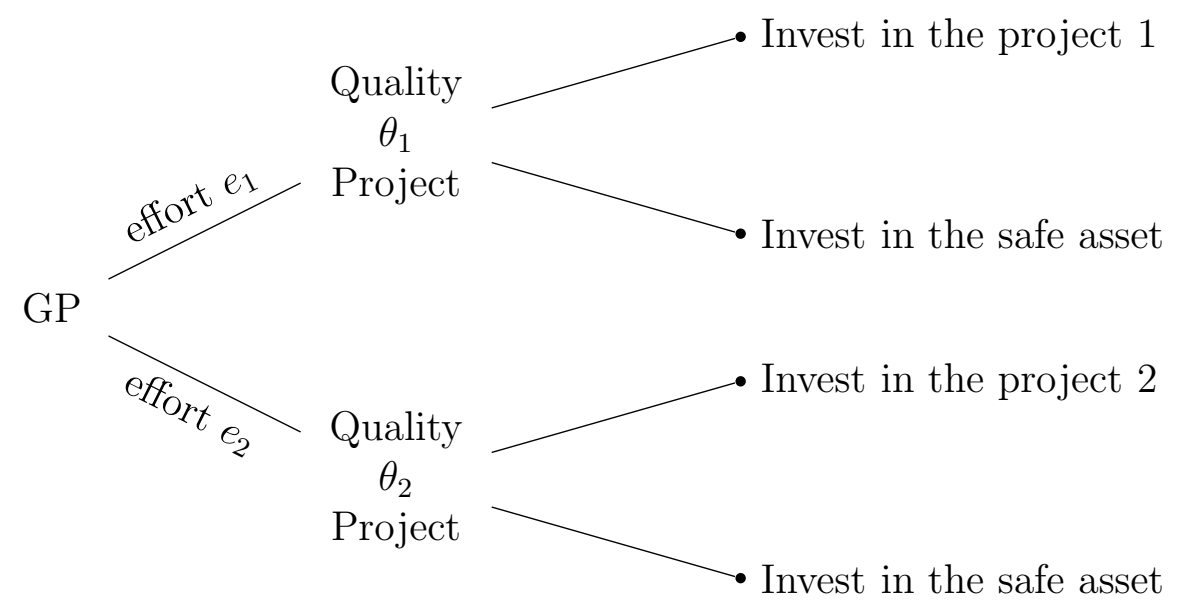

Figure 2: GP Problem

For simplicity and without loss of generality, assume that $p_{1} \geq p_{2}$. Note that in the optimum

$$
\begin{aligned}
& \rho \lambda_{\min } x+\left[\lambda_{1}-2 \rho \lambda_{\min }+\lambda_{2}\right] y+\left[1-\lambda_{1}-\lambda_{2}+\rho \lambda_{\min }\right] z \\
& =\max \left\{z+2 c, \lambda_{\max } y+\left(1-\lambda_{\max }\right) z+c\right\} .
\end{aligned}
$$

This is a typical phenomenon when dealing with moral hazard issues. This means that the expected payout to the GP is binding by the condition which induces exerting effort on both projects, otherwise the investor can lower payment in some states of the world without changing GP incentives. More formally, if equation (12) does not hold, the transformation $z \rightarrow z-\epsilon$ for small enough $\epsilon$ should violate the optimality conditions. Otherwise the LP can have a feasible contract with less expected payout to the GP. This means that $z=p_{1} y$, hence $z$ can not be reduced. Similarly $y \rightarrow y-\epsilon$ should violate the conditions as well, hence one gets $y=p_{1} x$. But then in any case $x \rightarrow x-\epsilon$ is possible because by the last two equalities we have $x>y \geq z$. Therefore the LP problem can be written as

$$
\begin{aligned}
& \min _{x, y, z} \alpha x+\beta y+\gamma z \\
& \alpha x+\beta y+\gamma z=\max \left\{z+2 c, \lambda_{\max } y+\left(1-\lambda_{\max }\right) z+c\right\} \\
& x \geq y \geq z \geq p_{1} y \geq p_{1}^{2} x
\end{aligned}
$$

where $(\alpha, \beta, \gamma)=\left(\rho \lambda_{\text {min }}, \lambda_{1}-2 \rho \lambda_{\text {min }}+\lambda_{2}, 1-\lambda_{1}-\lambda_{2}+\rho \lambda_{\text {min }}\right)$. In a similar vein, conditions that induce the choice of optimal investment strategies by the GP are binding as well. Therefore we have the following proposition. 
Proposition 2 (Optimal whole-portfolio contract) The optimal whole-portfolio contract satisfies

$$
z=p_{1} y=p_{1}^{2} x
$$

where

$$
z=\frac{2 c}{\beta \frac{1-p_{1}}{p_{1}}+\alpha \frac{1-p_{1}^{2}}{p_{1}^{2}}}
$$

if

$$
\rho \geq \rho^{*}=\frac{\lambda_{\max }-\lambda_{\min }}{\lambda_{\min }\left(\frac{1}{p_{1}}-1\right)}
$$

otherwise

$$
z=\frac{c}{\frac{\alpha\left(1-p_{1}^{2}\right)}{p_{1}^{2}}+\beta \frac{1-p_{1}}{p_{1}}-\lambda_{\max } \frac{1-p_{1}}{p_{1}}}
$$

Here is the intuition behind this proposition. When the investor writes the contract, he wants to choose the maximal value for $x$ to give the biggest incentive to the agent to exert effort. However, because of adverse selection, the prize for success cannot be too large, as it leads to inefficient investment decisions by the GP (investing in type $B$ projects). The maximum possible value for $x$ is $\frac{z}{p_{1} p_{2}}$ and maximum attains if and only if equation 13 holds. The two different regimes in the proposition correspond to the fact that the expected payout to the GP $(\alpha x+\beta y+\gamma z)$, becomes equal to $z+2 c$ or $\lambda_{\max } y+\left(1-\lambda_{\max }\right) z+c$. When the correlation is high, the GP either prefers to exert no effort or to exert effort on both projects because of high dependency between the success in both projects. Hence for high values of $\rho$,it is needed to pay enough to the GP such that the GP exerts any effort at all. This amount is $s(2 I)$ which is the reserve value for the GP. However, when the correlation is low, the payout should compensate for the lower level of inter-dependency between projects. So the payout should be high enough for the case success in both projects so that the GP does not find it beneficial to exert effort on only the easier project (corresponding to $\lambda_{\max }$ ).

Note that, as we mentioned, the security as defined here is increasing on the set of possible outcomes on the equilibrium path. However since $s_{G P}(R)=0$ and $s_{G P}(2 I)>0$, when $R>2 I$, optimal security is not increasing on all possible outcomes. This stems from the fact that the LP wants to push the GP to invest in only good projects and reserve the money if the project is bad. The non-monotonicity of the optimal security has been observed before in the literature, like Manso (2011). The mechanism in Manso (2011) which leads to this phenomena is the fact that the contract is written in a way to motivate experimentation by the agent. Hence the principal has to reward for failure so that the agent can take the risk. However, my setting has quite an opposite mechanism-the monotonicity arises because the GP wants to make the LP take less risk. For example, suppose the principal wants the 
agent to invest in a safe asset, and the agent goes and invests in a bad project instead. So if the payoff is high, it means that the agent deviated from the optimal strategy and as a result she gets punished.

The derivative of $\alpha \frac{1+p_{1}}{p_{1}}+\beta$ with respect to $\rho$ equals to

$$
\lambda_{\min }\left(\frac{1}{p_{1}}-1\right)>0
$$

hence the payout to the GP decreases as $\rho$ increases. Also note that the total payout of projects (which is $E\left[s_{G P}\right]+E\left[s_{L P}\right]$ ) equals to

$$
\alpha 2 R+\beta(R+I)+\gamma 2 I
$$

it has derivative (w.r.t $\rho$ )

$$
2 \lambda_{\min } R-2 \lambda_{\min }(R+I)+2 \lambda_{\min } I=0
$$

so total payout of projects is constant. Therefore we have the following

Proposition 3 (Comparing whole-portfolio with deal-by-deal) As the correlation $\rho$ increases, the expected payout to the GP decreases and the expected payout to the LP increases. Therefore, for admissible values $\left(\lambda_{1}, \lambda_{2}, p_{1}, p_{s}\right)$, there is $\rho^{* *}=\rho\left(\lambda_{1}, \lambda_{2}, p_{1}, p_{s}\right)$, such that for $\rho>\rho^{* *}$, whole-portfolio contracting is preferred by the LP and for $\rho<\rho^{* *}$ deal-bydeal contracting is preferred by the LP. In addition if $\rho \geq \rho^{*}$, whole-portfolio contracting is better for the LP ( equivalently $\rho^{* *} \leq \rho^{*}$ ).

The intuition for the proposition above comes from the fact that when the correlation between projects is higher, it becomes easier to encourage the GP to exert effort on both projects since success in one project increases the chance of success in the other one. As a result, the LP needs to pay less to motivate effort by the GP, hence the LP makes more profit because the total payout of projects is the same for all $\rho$. Since the deal-by-deal contract is independent from the correlation, from the monotonicity of payout with respect to correlation, we can see that if the LP prefers whole-portfolio contracting to deal-by-deal contracting for a given $\rho$, then as $\rho$ goes up it is still the case. As I pointed out in the introduction, this result observed empirically in Magro (2018). There the author shows that deal-by-deal compensation induces greater heterogeneity in portfolio investments. So the proposition above rationalizes this finding. In section 8 , I show that this result holds when a strong form of monotonicity is imposed on the security as well. 
Whole-portfolio contracting does not depend on $p_{2}$. This is because since $p_{1} \geq p_{2}$, the first project has more severe adverse selection problem compared to the second one. Therefore when information asymmetry constraint binds for the first project, it is already alleviate the adverse selection for the second project as well. Mathematically speaking when $z \geq p_{1} y$ then already we have $z>p_{2} y$ as well. When $\rho$ is large enough, projects are similar to each other and as we saw in Proposition 3, whole-portfolio contracting is more appealing for the LP. This is because, in this case, bundling efforts together gives the LP a big enough benefit that makes up for the loss which comes from having inefficient treatment of adverse selection (in contrast to the deal-by-deal contract which handles this issue efficiently). However for smaller values of $\rho$, the comparison of benefiting from bundling effort is smaller than the loss of sub-optimal handling of the information asymmetry problem. Recall that the expected payout to the GP in the deal-by-deal case is

$$
2 c+\Pi_{G P}=2 c+\sum_{i=1}^{2} \frac{p_{i} c}{\lambda_{i}\left(1-p_{i}\right)}
$$

which is increasing in $p_{2}$. Therefore the discussion above implies the following.

Proposition 4 (Asymmetry of information VS moral hazard) For given values $\left(\lambda_{1}, \lambda_{2}, p_{1}, \rho\right)$ , there is $p_{2}^{*}=p_{2}\left(\lambda_{1}, \lambda_{2}, p_{1}, \rho\right)$ such that for $p_{2}<p_{2}^{*}$ deal-by-deal contracting is better for the $L P$ and for $\left(p_{1} \geq\right) p_{2}>p_{2}^{*}$ whole-portfolio contracting. When $\rho>\rho^{* *}, p_{2}^{*}=0$.

While Proposition 3 resolves the comparison between whole-portfolio contracting and deal-by-deal in terms of correlation, Proposition 4 helps us to understand the comparison in terms of information asymmetry. Here is the intuition behind this statement. As mentioned before, the investor should take into account the loss of efficient handling of the adverse selection problem. The term $p_{1}-p_{2}$ measures the difference between the adverse selection issues that two projects are subject to. When $p_{1}-p_{2}$ is large (equivalently $p_{2}$ is small), the heterogeneity of asymmetry of information between the two projects is large. As a result, it is more efficient to have a deal-by-deal contract for better handling of this issue. Whereas for large $p_{2}$ (small $p_{1}-p_{2}$ ), the loss of efficiency on this issue is negligible, hence whole-portfolio contracting is better.

Whenever $p_{2}^{*}=0$, whole-portfolio contracting is dominant for the set of parameters given. When $\lambda_{\max }=\lambda_{1}$, then $p_{2}^{*}>0$ whenever $\rho<\rho^{*}$. However when the moral hazard problem is more severe in the first project as well (i.e $\lambda_{\max }=\lambda_{2}$ ), then for a larger set of $\rho$, whole-portfolio contracting is dominant. In this case, the investor uses the payout on the second project, which dominates the first in both moral hazard and information asymmetry 
aspects, as a prize to motivate GP to exert effort on the first project. From the proof of the Propositions [3, the equation that computes $p_{2}^{*}$ is given by (when $\rho<\rho^{*}$ )

$$
\begin{aligned}
& \lambda_{\max } \frac{z}{p_{1}}+\left(1-\lambda_{\max }\right) z+c=2 c+\sum_{i=1}^{2} \frac{p_{i} c}{\lambda_{i}\left(1-p_{i}\right)} \\
& z=\frac{c}{\frac{\alpha\left(1-p_{1}^{2}\right)}{p_{1}^{2}}+\beta \frac{1-p_{1}}{p_{1}}-\lambda_{\max } \frac{1-p_{1}}{p_{1}}}
\end{aligned}
$$

Relative to other variables, we have the following Proposition.

Proposition 5 (Comparative Statics) In the region $\rho>\rho^{*}$, expected payout to the GP is increasing in $p_{1}$ and is decreasing in $\lambda_{\max }$ and $\lambda_{\text {min }}$. For $\rho<\rho^{*}$, expected payout to the GP is increasing with respect to $p_{1}$ and $\lambda_{\max }$ and decreasing with respect to $\lambda_{\min }$.

Here is the intuition behind Proposition 5. In both regimes of $\rho$, when $p_{1}$ increases, the information asymmetry to be overcome by the LP worsens as the GP finds it more profitable to invest in bad projects. As a result, the expected payout to the GP increases when $p_{1}$ increases to compensate for information rent by the GP. With respect to $\lambda_{\min }$, as $\lambda_{\text {min }}$ increases, it becomes easier for the LP to motivate the GP to exert effort for the harder project, hence the expected payout is decreasing. However with respect to $\lambda_{\max }$, the relation to the payout depends on which regime $\rho$ is in. In the high correlation regime $\left(\rho>\rho^{*}\right)$, as we saw above, the GP has to compensate as much as needed to make the GP exert any effort. As in a classical moral hazard problem, when the task becomes easier the expected payout to the agent decreases. However, in the regime $\rho<\rho^{*}$, the LP has to compensate the GP for the strategy of exerting effort only on the easier project. This outside option's payout increases as $\lambda_{\max }$ increases, hence the LP has to compensate the GP more for not choosing this strategy.

\section{Non-Reputable GP}

Following Axelson et al (2009) and as mentioned in section 2, when the GP is not reputable, the LP cannot distinguish the non-reputable GPs from a fly-by-night operator (FNO). In this case the following assumption should be imposed on the securities to discourage FNOs from getting the investment outlay $2 I$ and enjoy the managerial fee $s(2 I)$ without exerting any effort..

- $s_{G P}(x)=0$ for $x \leq K$ where $K$ is the committed capital (FNO assumption). 
This assumption was first introduced in Axelson et al (2009) and has been used in subsequent works (for example Fang (2019)). Here I investigate how enforcing this condition can change the contract. Therefore, in essence we have a separating equilibrium in which a contract between the LP and a non-reputable GP satisfies the FNO assumption while a contract between the LP and a well-established (i.e., reputable) GP does not need this condition.

First note that with this assumption, first-best can not be implemented for a single project. This happens since $s_{G P}(I)=0$, the GP will invest in a project no matter the quality of the project as there is no reward for not investing in a type $B$ project, hence he implements strategy (3) discussed in section 2, The GP exerts effort if

$$
s^{F N O}(R) \geq \frac{c}{\lambda(1-p)}
$$

It is feasible to fund through this contract if and only if

$$
R-\frac{c}{\lambda(1-p)} \geq \frac{I}{\lambda+(1-\lambda) p}
$$

Here $\lambda+(1-\lambda) p$ is that chance of having return $R$. When the project is good (which has probability $\lambda$ ), return $R$ has probability 1 and if the project is bad (with probability $1-\lambda$ ), the probability of success is $p$. The profit made by the GP is

$$
\begin{aligned}
& \Pi_{G P}^{F N O}=(\lambda+(1-\lambda) p) \frac{c}{\lambda(1-p)}-c \\
& =\frac{p c}{\lambda(1-p)} .
\end{aligned}
$$

Comparing with equation (6), we can see that both reputable and non-reputable agents make the same profit. This comes from the fact that $s(I)=p s(R)$ for a reputable agent. Therefore a reputable GP gets the same payout as a non-reputable GP in the case of getting of a bad project and investing in the safe asset instead of making a bad investment, which is in the interest of the LP. Not surprisingly, the feasibility condition (15) is weaker compared to the case of a reputable agent which is (5) since here the investment strategy by the agent is not optimal. In the next part I find the optimal whole-portfolio contract and compare the results with that of section 3. If $p=0$, in a single project's contract, LP gets the whole surplus of the project so for the next part we assume $\max \left(p_{1}, p_{2}\right)>0$. 


\subsection{Whole-Portfolio Contracting With FNO}

In this part I find the optimal whole-portfolio contracting in the presence of the FNO assumption. By the FNO assumption, the contract satisfies

$$
s^{F N O}(I)=s^{F N O}(2 I)=0
$$

Since $s^{F N O}(2 I)=0$, it is impossible to motivate the GP to not invest in any bad project when both projects are bad (as the return to the GP will be zero in this case). So the best strategy that the LP can hope to achieve, similar to Axelson et al (2009), is the following

- The GP exerts effort on both projects.

- If at least one of the projects is good, the GP invests in only good projects (optimal choice).

- If both projects are bad, the GP invests in just one bad project.

As we can see, again $R$ is not the outcome of the optimal strategy, so in the optimal contract $s^{F N O}(R)=0$ holds as well. To induce (constrained) optimal choice of investment after efforts are exerted, assuming $p_{1} \geq p_{2}$, the LP should impose

$$
s^{F N O}(2 R) \geq s^{F N O}(R+I) \geq p_{1} s^{F N O}(2 R)
$$

Under these conditions, the GP invests only in good projects if any are available and invests in only one bad project if both projects are bad. With this strategy, the total payout to the GP becomes

$$
\begin{aligned}
& \rho \lambda_{\text {min }} s^{F N O}(2 R)+\left(\lambda_{1}-2 \rho \lambda_{\text {min }}+\lambda_{2}\right) s^{F N O}(R+I)+p_{1}\left(1-\lambda_{1}-\lambda_{2}+\rho \lambda_{\text {min }}\right) s^{F N O}(R+I) \\
& =\alpha x+\tilde{\beta} y
\end{aligned}
$$

where $(x, y)=\left(s^{F N O}(2 R), s^{F N O}(R+I)\right)$ and $(\alpha, \tilde{\beta})=\left(\rho \lambda_{\text {min }}, \lambda_{1}-2 \rho \lambda_{\text {min }}+\lambda_{2}+p_{1}\left(1-\lambda_{1}-\right.\right.$ $\left.\left.\lambda_{2}+\rho \lambda_{\text {min }}\right)\right)$. The only term which is different compared to the reputable agent is the last term. This comes from the fact that in the case of two bad projects, the GP invests in the project corresponded to $p_{1}$ and withhold money on the other one. Similar to what we had in Section 3, in order to induce effort on both projects, the contract should satisfy

$$
\alpha x+\tilde{\beta} y \geq p_{1} y+2 c, \theta_{i} y+c
$$


where $\theta_{i}=\lambda_{i}+p_{1}\left(1-\lambda_{i}\right)$. Therefore, the LP problem can be written as

$$
\begin{aligned}
& \min _{x, y} \alpha x+\tilde{\beta} y \\
& x \geq y \geq p_{1} x ; \quad \alpha x+\tilde{\beta} y \geq \max \left\{\theta_{\max } y+c, p_{1} y+2 c\right\}
\end{aligned}
$$

Like before, in the optimum the moral hazard constraint binds, hence

$$
\alpha x+\tilde{\beta} y=\max \left\{\theta_{\max } y+c, p_{1} y+2 c\right\}
$$

Similar to the reputable agent, adverse selection binds as well, and we have the following proposition.

Proposition 6 (Optimal whole-portfolio contract for non-reputable GP) In the optimal whole-portfolio contract we have

$$
y=p_{1} x
$$

If $\rho \geq \rho^{*}=\frac{\lambda_{\max }-\lambda_{\min }}{\lambda_{\min }\left(\frac{1}{p_{1}}-1\right)}$ then

$$
y=\frac{2 p_{1} c}{\alpha-p_{1}\left(p_{1}-\tilde{\beta}\right)}
$$

otherwise

$$
y=\frac{p_{1} c}{\alpha-p_{1}\left(\theta_{\max }-\tilde{\beta}\right)}
$$

Moreover $y=s^{F N O}(R+I)$ coincides with the payout to the GP in the reputable case $s(R+$ $I)=\frac{z}{p_{1}}$ from Proposition 2 .

With the same reasoning as in Proposition 2, two cases are associated with the fact that the expected payout to the GP $(\alpha x+\tilde{\beta} y)$ becomes $\theta_{\max } y+c$ or $p_{1} y+2 c$. In the non-reputable case, the total payout of both projects is

$$
2 \alpha R+\tilde{\beta}(R+I)+(1-\alpha-\tilde{\beta}) I
$$

The derivative with respect to $\rho$ of the total payout is

$$
\lambda_{\min }\left(p_{1} R-I\right)<0 .
$$

As we can see, on one hand the total payout of projects is decreasing with respect to the correlation between projects. Intuitively this is because the only scenario in which the investment decision is not optimal is when both projects are bad and the chance of this 
scenario is higher for higher correlation. On the other hand, the total payout to the GP is decreasing with respect to correlation as again it gets easier to motivate the GP to exert effort when the correlation goes up. As a result, the total payout to the investor is ambiguous with respect to correlation and depends on the relative magnitude of $p_{1} R-I$ and $c$. Here $p_{1} R-I$ measures the inefficiency associated with investing in the bad project with success chance $p_{1}$ (hence expected return $p_{1} R$ ) instead of investing in the safe asset (with return $I$ ). When $c$ is large enough, since the expected payout to the GP is proportional to $c$, the total payout to the GP decreases faster compared to the loss of inefficiency which is proportional to $p_{1} R-I$. As a result, by increasing $\rho$ the total payout to the LP increases as well. If $c$ is small enough, the reverse phenomenon happens and hence the total payout to the LP is decreasing with respect to $\rho$. Finally, in the middle range of $c$, total payout to the GP has an interior optimal correlation. Hence we do not have a monotonic relationship between the LP's payout and the correlation in the non-reputable case. However, we can show the following.

Proposition 7 (Reputable VS Non-reputable agent) Suppose for parameters $\left(\lambda_{1}, \lambda_{2}, p_{1}, p_{2}, \rho\right)$, the investor prefers whole-portfolio contracting when writing contracts with a generic (reputable) GP. This will also be the case when writing a contract with a non-reputable GP. In particular, if $\rho \geq \rho^{*}=\frac{\lambda_{\max }-\lambda_{\min }}{\lambda_{\min }\left(\frac{1}{p_{1}}-1\right)}$, then whole-portfolio contracting is dominant in the non-reputable case as well.

This proposition comes from the fact that when dealing with non-reputable agents, wholeportfolio contracting can help to improve the investment strategy. Hence the space of parameters in which whole-portfolio contracting is better for the LP is larger compared to the reputable case. As mentioned in the introduction, this result has been observed empirically in Huther et al (2020).

\section{Extension}

In this section, I consider various modifications of the model and how it can affect the results. I make some predictions/observations as well.

\subsection{No Asymmetric Information}

In the special case when $p_{1}=p_{2}=0$, as we mentioned in section 2 , there is no profitable bad option for the GP to invest in. Equivalently, there is no information asymmetry about the quality of projects between the GP and the LP as there is no possible profitable deviation. 
Under binary effort assumption, by equation (6) , the whole surplus of every project goes to the LP and hence the method of contracting is irrelevant in this setting. In this case when adverse selection is absent, since effort is binary, contract makes the GP indifferent between exerting effort and not exerting effort and hence the LP can get the first-best outcome. In order to analyze this important special case in depth, here I allow for different levels of effort to see how it affects the contract. Therefore for the purpose of this section, suppose with the variable cost $c_{i}\left(\lambda_{i}\right)$, the chance of getting a good $i$-project is $\lambda_{i}$. I assume $c_{i}(0)=c_{i}^{\prime}(0)=c_{i}^{\prime \prime}(0)=0$ and $c_{i}^{(3)}>0$. I first consider the general case and then restrict to the especial case $p_{1}=p_{2}=0$ which we are interested in. As before, the chance of success for a $i$-project of type $B$ is $p_{i}$. The first-best effort satisfies

$$
\max _{\lambda_{i}} \lambda_{i} R+\left(1-\lambda_{i}\right) I-c_{i}\left(\lambda_{i}\right)-I
$$

By FOC the optimal effort satisfies $c_{i}^{\prime}\left(\lambda_{i}^{F B}\right)=R-I$. Not surprisingly it is independent of $p_{i}$ as there is no adverse selection problem. I Assume $c_{i}^{\prime}(1)>R-I$ to make sure that $\lambda_{i}^{F B}<1$. Now suppose the contract $\left(s_{G P}(I), s_{G P}(R)\right)$ is offered to the GP. Similar to the binary case, the contract should satisfy

$$
s_{G P}(I) \geq p_{i} s_{G P}(R)
$$

to make sure that GP does not invest in the bad project. Once offered, the agent chooses effort $\lambda_{i}$ which is the solution to the problem

$$
\max _{\lambda_{i}} \lambda_{i} s_{G P}(R)+\left(1-\lambda_{i}\right) s_{G P}(I)-c_{i}\left(\lambda_{i}\right)
$$

FOC implies

$$
s_{G P}(R)-s_{G P}(I)=c_{i}^{\prime}\left(\lambda_{i}\right)
$$

in particular by decreasing $s_{G P}(I)$, the effort increases which is in the favor of LP (both lower payment and higher effort). Hence in the optimal $s_{G P}(I)=p_{i} s_{G P}(R)$ as contract should satisfies equation (17). This gives

$$
c_{i}^{\prime}\left(\lambda_{i}^{*}\right)=\left(1-p_{i}\right) s_{G P}(R)
$$

in the optimum. Once we have this, $\lambda_{i}$ is determined by solving

$$
\max _{\lambda_{i}} \lambda_{i}(R-I)-\frac{\lambda_{i} c_{i}^{\prime}\left(\lambda_{i}\right)+\left(1-\lambda_{i}\right) p_{i} c_{i}^{\prime}\left(\lambda_{i}\right)}{1-p_{i}}
$$


which comes from the LP problem

$$
\max _{\lambda_{i}} \lambda_{i}\left[R-s_{G P}(R)\right]+\left(1-\lambda_{i}\right)\left[I-p_{i} s_{G P}(R)\right]
$$

and equation (19). Specializing to the case $p_{i}=0$, one gets $c_{i}^{\prime}\left(\lambda_{i}^{*}\right)=s_{G P}(R)$. The equation to determine $\lambda_{i}$ becomes

$$
\max _{\lambda_{i}} \lambda_{i}(R-I)-\lambda_{i} c_{i}^{\prime}\left(\lambda_{i}\right)
$$

and FOC gives

$$
R-I=c_{i}^{\prime}\left(\lambda_{i}^{*}\right)+\lambda_{i} c^{\prime \prime}{ }_{i}\left(\lambda_{i}^{*}\right) .
$$

When comparing the second-best effort with first best (equation (18)), the extra term $\lambda_{i} c_{i}^{\prime \prime}\left(\lambda_{i}\right)$ measures the moral hazard issue and it reduces the effort by the agent. So in this more flexible setting, first-best is not contactable even without adverse selection. The expected payout from one project to the LP is

$$
\lambda_{i}\left(R-c_{i}^{\prime}\left(\lambda_{i}\right)\right)+\left(1-\lambda_{i}\right) I-I
$$

which can be written as

$$
\begin{aligned}
& \lambda_{i}\left(R-I-c_{i}^{\prime}\left(\lambda_{i}\right)\right) \\
& =\lambda_{i}^{2} c_{i}^{\prime \prime}\left(\lambda_{i}\right) .
\end{aligned}
$$

The expected payout to the GP is $\lambda_{i} c_{i}^{\prime}\left(\lambda_{i}\right)$ and $\lambda_{i} c_{i}^{\prime}\left(\lambda_{i}\right)-c_{i}\left(\lambda_{i}\right)>0$ is the expected profit for the the GP.

For the whole-portfolio contracting, I consider a simple whole-portfolio contract which pays agent only when the outcome is $2 R$. In this case, the GP problem is

$$
\max _{\lambda_{1}, \lambda_{2}} \lambda_{1} \lambda_{2} s_{G P}(2 R)-c_{1}\left(\lambda_{1}\right)-c_{2}\left(\lambda_{2}\right)
$$

which gives

$$
\begin{aligned}
& c_{2}^{\prime}\left(\lambda_{2}\right)=\lambda_{1} s_{G P}(2 R) \\
& c_{1}^{\prime}\left(\lambda_{1}\right)=\lambda_{2} s_{G P}(2 R)
\end{aligned}
$$

Once this, the LP problem is to determine $s_{G P}(2 R)$ to maximize the expected revenue which 
is

$$
\max _{s_{G P}(2 R)} \lambda_{1} \lambda_{2}\left(2 R-s_{G P}(2 R)\right)+\left[\lambda_{1}\left(1-\lambda_{2}\right)+\lambda_{2}\left(1-\lambda_{1}\right)\right](R+I)+\left(1-\lambda_{1}\right)\left(1-\lambda_{2}\right) 2 I
$$

where $\lambda_{1}, \lambda_{2}$ satisfy GP's optimality equations. We have the following proposition which gives answer for a wide class of cost functions.

Proposition 8 (No asymmetric information case) If $c_{1}=a \lambda^{m}$ and $c_{2}=b \lambda^{m}$ for $m>$ 2 and $a, b>0$, then whole-portfolio contracting is better for the LP compared to deal-by-deal contract.

The intuition behind the proposition is simple. When there is no asymmetry of information, it is better that contract motivates effort as easily as possible. Tying outcomes together can provide a bigger incentive relative to contracting on projects in deal-by-deal basis. This proposition shed light on the fact that in settings where the investor and agent have same information about the quality of projects, the whole-portfolio contracting is dominant. This includes hedge-funds, mutual-bonds or other contracts on public equities.

\subsection{Uninformed Investor}

In this part, I consider the case in which investor is not informed about the correlation. Other than this, I assume the same setup as in the main model. Since in the deal-by-deal contract correlation has no effect on the outcome, I only focus on the whole-portfolio contracting. Assume that the GP can privately and strategically choose the correlation $\rho$ in the interval $\left[\rho_{1}, \rho_{2}\right]$. While the interval is common knowledge, the LP does not observe $\rho$ directly. The case of informed investor is a special case when $\rho_{1}=\rho_{2}=\rho$. Since investment compatibility conditions are independent from $\rho$, as in the informed case, optimal contract satisfies

$$
\begin{aligned}
& z \geq p_{1} y, p_{2} y, p_{1} p_{2} x \\
& y \geq z, p_{1} x, p_{2} x \\
& x \geq z, y
\end{aligned}
$$

where variables are as in equation (11). As before, assume $p_{1} \geq p_{2}$. The expected payout to the GP from choosing $\rho$ is

$$
\rho \lambda_{\min } x+\left[\lambda_{1}-2 \rho \lambda_{\min }+\lambda_{2}\right] y+\left[1-\lambda_{1}-\lambda_{2}+\rho \lambda_{\min }\right] z
$$


Derivative with respect to $\rho$ of the expression above is

$$
\lambda_{\min }(x-2 y+z) .
$$

There are two possible scenarios for the GP to choose the correlation. If $x>2 y-z$, the payout for two successful exits are relatively high hence GP wants to maximize the chance of having two successful exits. Therefore GP chooses the highest possible correlation i.e $\rho=\rho_{2}$. In contrary, if $y$ is relatively high $(2 y>x-z)$, then it is more profitable for GP to have only one successful exit. This event has the highest chance when $\rho$ is smallest which is $\rho=\rho_{1}$. Now suppose $(x, y, z)$ has the form of $\left(\frac{z}{p_{1}^{2}}, \frac{z}{p_{1}}, z\right)$ which is the same as in the optimal contract with informed investor from Proposition 2. In this case, the derivative with respect to $\rho$ of the payout to GP becomes

$$
\lambda_{\min } z\left(\frac{1}{p_{1}^{2}}-\frac{2}{p_{1}}+1\right)=\lambda_{\min } z\left(\frac{1}{p_{1}}-1\right)^{2}>0 .
$$

Therefore as argued above, GP chooses the highest value of $\rho$ which is $\rho_{2}$. As we saw in the informed problem, when $\rho$ goes up, $E\left[s_{L P}\right]$ goes up as well in the optimal informed contract. Therefore LP optimally offers the contract $\left(\frac{z^{*}}{p_{1}^{2}}, \frac{z^{*}}{p_{1}}, z^{*}\right)$ where $z^{*}=z^{*}\left(\rho_{2}\right)$ is the management fee in the optimal contract for the correlation $\rho_{2}$ from Proposition 2. GP optimally chooses $\rho_{2}$ as well from discussion above. Therefore if for $\rho=\rho_{2}$, LP prefers whole-portfolio contracting to deal-by-deal, then the contract above is offered. Otherwise deal-by-deal contract is offered. In summary we have

Proposition 9 (Uninformed Investor) Suppose GP can privately chooses $\rho$ in the interval $\left[\rho_{1}, \rho_{2}\right]$.Then

- If $\rho_{2}<\rho^{* *}$, a deal-by-deal contract is offered to GP.

- If $\rho_{2} \geq \rho^{* *}$, a whole-portfolio contract associated to $\rho=\rho_{2}$ is offered to GP and GP chooses $\rho=\rho_{2}$ optimally.

\subsection{Conditional Contract}

In this part, I consider conditional contracting which means that the payout of the contract is a function of the outcome of each project. This definition contains both deal-by-deal contracting as well as whole-portfolio contracting as special cases. When the payout of projects are $2 I$ or $2 R$, it corresponds uniquely to two bad or good projects respectively. However, there are two possible ways to get the outcome $R+I$. When the first project is 
type $G$ or when the second one is and the other one is type $B$. Unlike the whole-portfolio and similar to deal-by-deal, when the contract is fully conditional, total payout to GP can be different in these two cases. Therefore take $y_{1}$ and $y_{2}$ as possible payouts to GP where $y_{1}$ is $s(R+I)$ when the first project is successful and $y_{2}$ is that of when the second one is type $G$. Set $x=s(2 R)$ and $z=s(2 I)$ as in the whole-portfolio contracting. Also recall that from equation (10), we have $s(I)=s(R)=0$. Similar to whole-portfolio contracting, in the optimal contract, payouts satisfy

$$
\begin{aligned}
& z \geq p_{1} p_{2} x, p_{1} y_{1}, p_{2} y_{2} \\
& y_{1} \geq z, p_{2} x \\
& y_{2} \geq z, p_{1} x \\
& x \geq z, y_{1}, y_{2}
\end{aligned}
$$

When comparing these to analogues inequalities in the whole-portfolio contracting, we see that there is efficiency gain. recall that in the whole-portfolio contracting, $z$ should be bigger than both $p_{1} y$ and $p_{2} y$ since there is no difference between payouts to GP when the return is $R+I$ and the first project is successful or the return is $R+I$ and the second project is successful. As a result LP should overcompensate GP to cover both cases and this causes some inefficiency when compared to conditional contracting. The same phenomena happens when comparing $y$ and $x . y$ should be bigger than both $p_{1} x$ and $p_{2} x$ while in the conditional contract there are two different values $y_{1}$ and $y_{2}$ instead of single payout $y$. Assume $y_{\min }$ and $y_{\max }$ are the corresponding payouts for when the project with $\lambda_{\min }$ or $\lambda_{\max }$ succeed respectively. To motivate GP to exert effort on both projects (moral hazard problem), the contract should satisfy

$$
\begin{aligned}
& \rho \lambda_{\min } x+\left(\lambda_{\max }-\rho \lambda_{\min }\right) y_{\max }+(1-\rho) \lambda_{\min } y_{\min }+\left(1-\lambda_{1}-\lambda_{2}+\rho \lambda_{\min }\right) z \\
& \geq z+2 c, \lambda_{1} y_{1}+\left(1-\lambda_{1}\right) z+c, \lambda_{2} y_{2}+\left(1-\lambda_{2}\right) z+c
\end{aligned}
$$

So LP problem is

$$
\begin{aligned}
& \min _{x, y, z} \alpha x+\beta_{1} y_{1}+\beta_{2} y_{2}+\gamma z \\
& \alpha x+\beta_{1} y_{1}+\beta_{2} y_{2}+\gamma z \geq z+2 c, \lambda_{i} y_{i}+\left(1-\lambda_{i}\right) z+c \\
& x \geq y_{i} \geq z \geq p_{i} y_{i} \geq p_{1} p_{2} x
\end{aligned}
$$


where $\left(\alpha, \beta_{1}, \beta_{2}, \gamma\right)=\left(\rho \lambda_{\text {min }}, \lambda_{\text {max }}-\rho \lambda_{\text {min }},(1-\rho) \lambda_{\text {min }}, 1-\lambda_{1}-\lambda_{2}+\rho \lambda_{\text {min }}\right)$. With similar reasoning as in the whole-portfolio contracting, in the optimum

$$
\alpha x+\beta_{1} y_{1}+\beta_{2} y_{2}+\gamma z=\max \left\{z+2 c, \lambda_{i} y_{i}+\left(1-\lambda_{i}\right) z+c\right\}
$$

Similar to Proposition 2 we have the following.

Proposition 10 (Optimal Conditional Contract) In the optimal contract,

$$
z=p_{1} y_{1}=p_{2} y_{2}=p_{1} p_{2} x
$$

$z$ is determined such that equation (20) is satisfied.

As in the whole-portfolio contracting, the inequalities which are dealing with adverse selection issue are binding as well. It is worth mentioning that when $p_{1}=p_{2}$, we get $y_{1}=y_{2}$ even in the case that $\lambda_{1} \neq \lambda_{2}$, hence the contract reduces to a whole-portfolio contract. This is because the variation in $\lambda_{\mathrm{s}}$ affect the moral hazard problem and has no bearing on the asymmetric information issue which rises after exerting effort on projects. In fact when we compare whole-portfolio contracting with the conditional contracting for the loss of efficiency for LP, we have (recall that $p_{2}<p_{1}$ )

Proposition 11 (Efficiency loss for whole-portfolio contracting) The profit made by $G P$ in the conditional contracting decreases as $p_{2}\left(\leq p_{1}\right)$ increases. When $p_{2}=p_{1}$, the profit equals the profit made in the whole-portfolio contracting.

Here is the intuition behind statement above. . As we mentioned in the discussion after proposition 4, $p_{1}-p_{2}$ measures the difference between adverse selection that problems are subject to. When this measure is zero, handling of the problem for one project, efficiently takes care of the other project as well hence whole-portfolio contracting becomes the best conditional contract. As this measure grows, whole-portfolio contracting becomes less and less efficient and GP can extract more rent on the projects.

\subsection{Bargaining Power}

In this part, I consider what happens when GP has the bargaining power for writing the contract. For the moment I assume that the offered contract has to be incentive compatible to induce the GP to choose the optimal strategy (justify it at the end). For reputable GP, 
the problem becomes

$$
\begin{aligned}
& \max _{x, y, z} \alpha x+\beta y+\gamma z \\
& x \geq y \geq z \geq p_{1} y \geq p_{1}^{2} x ; \quad \alpha x+\beta y+\gamma z \geq z+2 c, \lambda_{\max } y+\left(1-\lambda_{\max }\right) z+c \\
& E\left[s_{L P}\right] \geq 2 I
\end{aligned}
$$

The last inequality is the participation constraint for the LP. Here notation are the same as in subsection 3.2. Not surprisingly, in the optimum, the equality $E\left[s_{L P}\right]=2 I$ happens otherwise the contract can be altered in the GP's favor without violating participation constraint by LP (for example by increasing $x$ ). Hence unlike investor problem we do not have a unique contract and the contract only needs to satisfy the incentive compatibility equations. Also, as we saw in subsection $\left[3.2, E\left[s_{L P}\right]+E\left[s_{G P}\right]\right.$ is independent of $\rho$. Therefore, investor breaks even in the optimum and the contract is not unique. To justify the imposing of the incentive compatibility, note that in any feasible contract $E\left[s_{L P}\right] \geq 2 I$ is required. Also the optimal investment strategy guarantees the maximum possible payout of the projects. Hence imposing them does not reduce the profit by GP.

This result is not surprising as when GP has the market power, since she is the party who takes the action and also observes the quality of the project, she can extract all the rent from projects. So in the presence of the market power by GP, the method of contracting or correlation does not play a role.

When GP is not reputable hence FNO is imposed, take $E\left[s_{G P}\right]=\alpha x+\tilde{\beta} y$ as in section 4 and then the GP problem becomes

$$
\begin{aligned}
& \max _{x, y} \alpha x+\tilde{\beta} y \\
& x \geq y \geq p_{1} x ; \quad \alpha x+\tilde{\beta} y \geq \max \left\{\theta_{\max } y+c, p_{1} y+2 c\right\} \\
& E\left[s_{L P}\right] \geq 2 I
\end{aligned}
$$

Similar to the case of reputable GP, investor breaks even and he is not concerned about the correlation. In addition, if GP can choose the correlation as well, she maximizes the surplus of the project. So as we saw in the section 4 , total payout is decreasing in $\rho$ so she chooses $\rho=0$ in this case. Since even for $\rho=1$, total payout of projects is more than deal-by-deal contract, when GP has market power she chooses whole-portfolio contracting. This stems from the fact that the whole-portfolio contracting persuade GP to have a better investment strategy. Hence in summary we get

Proposition 12 When GP has the bargaining power, for reputable GP there is no difference 
between deal-by-deal and whole-portfolio contracting. For non-reputable GP whole-portfolio contracting is preferred for all values of $\rho$. In addition the contract is not unique and only needs to satisfy the IC by GP as well as PC by LP.

\section{Conclusion}

In this paper, I proposed a framework to study the scheme of payment in LPA. Unlike usual contracts which only determine the amount of payment for a given return, since GP and LP write a contract on a portfolio, the method of payment is also of vital importance. I compared the main two methods of payments which are the deal-by-deal and the whole-portfolio. Within my setting, I showed that the whole-portfolio contracting is more prevalent when the correlation of investment companies is high or when the reputation of GP is low. Previously documented findings support these result.

In addition, I make some predictions which can guide future studies. For example the informativeness of the investor can also affect the method of contracting and hence the portfolio as well. More informed investors tend to have more deal-by-deal contract and a diverse portfolio while less informed ones have a narrower range of investment and more wholeportfolio contracting. Also when underlying assets are public, whole-portfolio contracting is the typical method of payment which is used.

\section{References}

[1] Axelson, Ulf, Per Stromberg, and Michael S. Weisbach, 2009. Why Are Buyouts Levered? The Financial Structure of Private Equity Funds. The Journal of Finance LXIV, $1549-1582$.

[2] Chung, Ji-Woong, Berk A. Sensoy, Lea Stern, and Michael S. Weisbach, 2012. Pay for Performance from Future Fund Flows: The Case of Private Equity. The Review of Financial Studies 25:3259-3304.

[3] Cumming Douglas and Sofia Johan, 2009. Venture Capital and Private Equity Contracting, Elsevier.

[4] Diamond, Douglas, 1984. Financial Intermediation and Delegated Monitoring," Review of Economic Studies, 51, 393-414. 
[5] Fang, Dawei, 2019. Dry powder and short fuses: Private equity funds in emerging markets. Journal of Corporate Finance 59, 48-71.

[6] Fulghieri, Paolo and Merih Sevilir, 2009. Size and Focus of a Venture Capitalist's Portfolio. The Review of Financial Studies 22, 4644-4680.

[7] Gompers, Paul and Josh Lerner, 1999. An analysis of compensation in the U.S. venture capital partnership. Journal of Financial Economics 51, 3-44.

[8] He, Zhiguo, Bin Wei, Jianfeng Yu, Feng Gao, 2017. Optimal Long-Term Contracting with Learning. The Review of Financial Studies 30, 2006-2065.

[9] Hüther, Niklas, David T. Robinson, Sönke Sievers, and Thomas Hartmann-Wendels, 2019. Paying for Performance in Private Equity: Evidence from Venture Capital Partnerships. Management Science, forthcoming.

[10] Inderst, Roman, Holger M. Mueller, and Felix M"unnich, 2007. Financing a Portfolio of Projects. The Review of Financial Studies 20, 1289-1325.

[11] Koskinen, Yrjo“, Michael J. Rebello, and Jun Wang, 2014. Private Information and Bargaining Power in Venture Capital Financing. Journal of Economics and Management Strategy 23, 743-775.

[12] Manso, Gustavo, 2011. Motivating Innovation. The Journal of Finance LXVI, 18231869.

[13] Magro, Joao. 2018. Deal-by-deal compensation structures and portfolio diversification. Working paper.

[14] Metrick, Andrew and Ayako Yasuda, 2010. The Economics of Private Equity Funds. The Review of Financial Studies 23, 2303-2341.

[15] Miao, Jianjun and Alejandro Rivera, 2016. Robust Contracts in Continuous Time. Econometrica 84, 1405-1440.

[16] Nachman, David and Thomas Noe, 1994. Optimal design of securities under asymmetric information. The Review of Financial Studies 7, 1-44.

[17] Pourbabaee, Farzad, 2020. Robust experimentation in the continuous time bandit problem. Economic Theory, 1-31.

[18] Repullo, Rafael and Javier Suarez, 2004. Venture Capital Finance: A Security Design Approach. Review of Finance 8, 75-108. 
[19] Robinson, David and Berk A Sensoy, 2003. Do private equity fund managers earn their fees? compensation, ownership, and cash flow performance. The Review of Financial Studies 26, 2760 - 2797.

\section{Appendix A: Proofs}

\subsection{Proof of Proposition 10}

Let's recall the equations which LP should consider to design the security. We have

$$
\begin{aligned}
& z \geq p_{1} p_{2} x, p_{1} y_{1}, p_{2} y_{2} \\
& y_{1} \geq z, p_{2} x \\
& y_{2} \geq z, p_{1} x \\
& x \geq z, y_{1}, y_{2}
\end{aligned}
$$

where as defined before, $(x, z)=(s(2 R), s(2 I))$ and $y_{i}$ is the payout to GP when project $i$ is successful and project $3-i$ is not. As we saw, LP problem is

$$
\begin{aligned}
& \min _{x, y, z} \alpha x+\beta_{1} y_{1}+\beta_{2} y_{2}+\gamma z \\
& \alpha x+\beta_{1} y_{1}+\beta_{2} y_{2}+\gamma z \geq z+2 c, \lambda_{i} y_{i}+\left(1-\lambda_{i}\right) z+c \\
& x \geq y_{i} \geq z \geq p_{i} y_{i} \geq p_{1} p_{2} x
\end{aligned}
$$

where $\left(\alpha, \beta_{1}, \beta_{2}, \gamma\right)=\left(\lambda_{1} \lambda_{2}, \lambda_{1}-\rho \lambda_{2},(1-\rho) \lambda_{2}, 1-\lambda_{1}-\lambda_{2}+\rho \lambda_{2}\right)$. In the discussion before the proposition, we showed that

$$
\alpha x+\beta_{1} y_{1}+\beta_{2} y_{2}+\gamma z=\max \left\{z+2 c, \lambda_{i} y_{i}+\left(1-\lambda_{i}\right) z+c\right\}
$$

Now we claim that, in the optimum either $z=p_{1} y_{1}$ or $z=p_{2} y_{2}$. This happens since if $z$ can be reduced, the coefficient of $z$ on the LHS which is $\gamma=1-\lambda_{1}-\lambda_{2}+\rho \lambda_{2}$ is less than (or equal to if $\rho=1$ ) the minimum of coefficients on the RHS of equation (22) which is $\min \left(1-\lambda_{1}, 1-\lambda_{2}\right)$. Having this, assume that $z=p_{1} y_{1} \geq p_{2} y_{2}$. Consider several cases

- First assume that the maximum on the RHS of equation (22) happens at $z+2 c$. Then we get

$$
\alpha x=z\left(1-\frac{\beta_{1}}{p_{1}}-\gamma\right)-\beta_{2} y_{2}+2 c .
$$


After multiplying by $p_{1} p_{2}$ and using the facts that $y_{2} \leq \frac{z}{p_{2}}, x \leq \frac{z}{p_{1} p_{2}}$ we have

$$
\begin{aligned}
& z\left[\alpha\left(1-p_{1} p_{2}\right)+p_{2} \beta_{1}\left(1-p_{1}\right)+p_{1} \beta_{2}\left(1-p_{2}\right)\right] \\
& \geq 2 p_{1} p_{2} c
\end{aligned}
$$

with strict inequality if either $y_{2}<\frac{z}{p_{2}}$ or $x<\frac{z}{p_{1} p_{2}}$. This gives a lower bound for $z$. In the optimum the inequality becomes equality (as LP looks for the minimum payment to GP) hence we get $z=p_{1} y_{1}=p_{2} y_{2}=p_{1} p_{2} x$.

- Now assume that maximum happens at $\lambda_{1} y_{1}+\left(1-\lambda_{1}\right) z+c$. Then similar to the previous case, we can write

$$
\alpha x=\lambda_{1} \frac{z}{p_{1}}+\left(1-\lambda_{1}\right) z-\beta_{1} \frac{z}{p_{1}}-\beta_{2} y_{2}-\gamma z+c
$$

Again inequalities as in the previous case gives us a lower bound for $z$ which is binding in the optimum hence we get the same relationship between variables.

- Finally assume that maximum happens at $\lambda_{2} y_{2}+\left(1-\lambda_{2}\right) z+c$ and assume this is strictly bigger than other two terms $\left(z+2 c\right.$ and $\left.\lambda_{1} y_{1}+\left(1-\lambda_{1}\right) z+c\right)$. If $z=p_{1} y_{1}=p_{2} y_{2}$, then the same reasoning as in the previous case works. So assume that $z=p_{1} y_{1}>p_{2} y_{2} \geq p_{1} p_{2} x$. Now reduce $z$ by $\epsilon$ and $y_{1}$ by $\frac{\epsilon}{p_{1}}$ and change $x$ accordingly to $x^{\prime}$ such that

$$
\alpha x^{\prime}+\beta_{1}\left(y_{1}-\frac{\epsilon}{p_{1}}\right)+\beta_{2} y_{2}+\gamma(z-\epsilon)=\lambda_{2} y_{2}+\left(1-\lambda_{2}\right)(z-\epsilon)+c
$$

If this change is permissible, then the new contract $\left(x^{\prime}, y_{1}-\frac{\epsilon}{p_{1}}, y_{2}, z-\epsilon\right)$ is strictly better for LP. So it would not be permissible. If $x^{\prime}>x$, this means that we should have had $p_{1} x=y_{2}$ so that an increase in $x$ is not permissible. However in this case, the original equation for the expected payout to GP, becomes

$$
\alpha\left(x-y_{2}\right)=\alpha x\left(1-p_{1}\right)=z\left(1-\frac{\beta_{1}}{p_{1}}-\gamma-\lambda_{2}\right)+c
$$

So

$$
\alpha \frac{z}{p_{1} p_{2}}\left(1-p_{1}\right) \geq z\left(1-\frac{\beta}{p_{1}}-\gamma-\lambda_{2}\right)+c
$$

and again in the optimum this should be equality hence $z=p_{1} p_{2} x$ which proves the claim. If $x^{\prime}<x$, then either $x=y_{2}$ or $x=y_{1}$. But these contracts can not be optimal since reducing all the payouts $z, y_{1}$ and $y_{2}$ and increasing $x$ is allowed here which improves payout to LP. 


\subsection{Proof of Proposition 2 and 3}

Let's recall the LP problem.

$$
\begin{aligned}
& \min _{x, y, z} \alpha x+\beta y+\gamma z \\
& \alpha x+\beta y+\gamma z \geq z+2 c, \lambda_{\max } y+\left(1-\lambda_{\max }\right) z+c \\
& x \geq y \geq z \geq p_{1} y \geq p_{1}^{2} x
\end{aligned}
$$

where $(\alpha, \beta, \gamma)=\left(\rho \lambda_{\text {min }}, \lambda_{1}-2 \rho \lambda_{\text {min }}+\lambda_{2}, 1-\lambda_{1}-\lambda_{2}+\rho \lambda_{\text {min }}\right)$. As in the case of Proposition 10, we make two preliminary observations.

1. In the optimum, we have $\alpha x+\beta y+\gamma z=\max \left\{z+2 c, \lambda_{\max } y+\left(1-\lambda_{\max }\right) z+c\right\}$. Suppose not. Then the transformation $z \rightarrow z-\epsilon$ for small enough $\epsilon$, should violate the conditions of the LP problem otherwise reducing $z$ improves the payout to LP. This means $z=p_{1} y$. But then $y \rightarrow y-\epsilon$ should violate the conditions so similarly we get $y=p_{1} x$ as well. But then $x \rightarrow x-\epsilon$ is legitimate because by $z=p_{1} y=p_{1}^{2} x$ we have $x>y>z$.

2. In the optimum, $z=p_{1} y$. This comes from the fact that in the LP problem $\gamma=$ $1-\lambda_{1}-\lambda_{2}+\rho \lambda_{\min } \leq 1-\lambda_{1}, 1-\lambda_{2}$. So if the move $z \rightarrow z-\epsilon$ is allowed, the subtraction on the LHS which is $\gamma \epsilon$ is less than the subtraction on the RHS which is either $\epsilon$ or $\left(1-\lambda_{\max }\right) \epsilon$.

By point 1 above, the expected payout to GP will be $\max \left\{z+2 c, \lambda_{\max } y+\left(1-\lambda_{\max }\right) z+c\right\}$. This function is increasing in $z$, equals to $z+2 c$ for $z \leq \frac{p_{1} c}{\lambda_{\max }\left(1-p_{1}\right)}$ (since $z=p_{1} y$ ) and it is $\lambda_{\max } y+\left(1-\lambda_{\max }\right) z+c$ otherwise. Because the payout is increasing in $z$, GP searches for a feasible contract with least amount of $z$. First we see when LP is able to write a contract with $z \leq \frac{p_{1} c}{\lambda_{\max }\left(1-p_{1}\right)}$. Here is how the contract is designed. As we saw, in this range of $z$,

$$
\alpha x+\beta y+\gamma z=\max \left\{z+2 c, \lambda_{\max } y+\left(1-\lambda_{1}\right) z+c\right\}=z+2 c
$$

therefore

$$
x=\frac{z\left(1-\gamma-\frac{\beta}{p_{1}}\right)+2 c}{\alpha}
$$

hence GP should solve

$\min z$

$x \geq \frac{z}{p_{1}} \geq p_{1} x \quad z \leq \frac{p_{1} c}{\lambda_{\max }\left(1-p_{1}\right)}$ 
given $x$ as in the equation (23) $\cdot p_{1} x \geq z$ implies

$$
2 p_{1} c \geq\left(1-p_{1}\right)(\alpha+\beta) z
$$

which implies

$$
z \leq \frac{2 p_{1} c}{(\alpha+\beta)\left(1-p_{1}\right)}
$$

On the other hand $z \geq p_{1}^{2} x$, implies

$$
\left[\beta \frac{1-p_{1}}{p_{1}}+\alpha \frac{1-p_{1}^{2}}{p_{1}^{2}}\right] z \geq 2 c
$$

which gives

$$
z \geq \frac{2 c}{\beta \frac{1-p_{1}}{p_{1}}+\alpha \frac{1-p_{1}^{2}}{p_{1}^{2}}}
$$

Since

$$
\frac{2 c}{\beta \frac{1-p_{1}}{p_{1}}+\alpha \frac{1-p_{1}^{2}}{p_{1}^{2}}}<\frac{2 p_{1} c}{(\alpha+\beta)\left(1-p_{1}\right)}
$$

one needs to compare the lower bound on the $z$ from equation (24) with the initial condition $z \leq \frac{p_{1} c}{\lambda_{\max }\left(1-p_{1}\right)}$. This gives us that there is answer in this region if and only if

$$
\left[\beta \frac{1-p_{1}}{p_{1}}+\alpha \frac{1-p_{1}^{2}}{p_{1}^{2}}\right] \geq \frac{2 \lambda_{\max }\left(1-p_{1}\right)}{p_{1}}
$$

which simplifies to

$$
\beta+\alpha \frac{1+p_{1}}{p_{1}} \geq 2 \lambda_{\max }
$$

Substituting $\alpha$ and $\beta$ gives us that this happens if and only if

$$
\rho \geq \frac{\lambda_{\max }-\lambda_{\min }}{\lambda_{\min }\left(\frac{1}{p_{1}}-1\right)}
$$

In this case the contract is written with $z$ as given by equality in the equation (24) which is the minimal $z$ hence $z=p_{1} y=p_{1}^{2} x$. Now consider the case $\rho<\frac{\lambda_{\max }-\lambda_{\min }}{\lambda_{\min }\left(\frac{1}{p_{1}}-1\right)}$. The expected payout to GP then has the form $\lambda_{\max } y+\left(1-\lambda_{\max }\right) z+c$ for some $z>\frac{p_{1} c}{\lambda_{\max }\left(1-p_{1}\right)}$. In this case we get

$$
\alpha x+\beta y+\gamma z=\lambda_{\max } y+\left(1-\lambda_{\max }\right) z+c=\left(\frac{\lambda_{\max }}{p_{1}}+1-\lambda_{\max }\right) z+c
$$


hence

$$
x=\frac{\left(\frac{\lambda_{\max }}{p_{1}}+1-\lambda_{\max }-\frac{\beta}{p_{1}}-\gamma\right) z+c}{\alpha}
$$

Similar to the previous case, the optimization becomes

$$
\begin{aligned}
& \min z \\
& x \geq \frac{z}{p_{1}} \geq p_{1} x \quad z \geq \frac{p_{1} c}{\lambda_{\max }\left(1-p_{1}\right)}
\end{aligned}
$$

$p_{1} x \geq z$ implies

$$
\left[\lambda_{\max }+p_{1}-p_{1} \lambda_{\max }-\beta-p_{1} \gamma\right] z+p_{1} c \geq \alpha z
$$

which is

$$
p_{1} c \geq\left(\alpha+\beta-\lambda_{\max }\right)\left(1-p_{1}\right) z
$$

We have $\alpha+\beta-\lambda_{\max }=\lambda_{\min }(1-\rho)$

$$
z \leq \frac{p_{1} c}{\left(1-p_{1}\right) \lambda_{\min }(1-\rho)}
$$

Finally $z \geq p_{1}^{2} x$ implies

$$
\left(\frac{\lambda_{\max }}{p_{1}}+1-\lambda_{\max }-\frac{\beta}{p_{1}}-\gamma\right) z+c \leq \frac{\alpha z}{p_{1}^{2}}
$$

which implies

$$
c \leq\left[\frac{\alpha}{p_{1}^{2}}-\left(\frac{\lambda_{\max }}{p_{1}}+1-\lambda_{\max }-\frac{\beta}{p_{1}}-\gamma\right)\right] z
$$

We can write this as

$$
c \leq\left[\frac{\alpha\left(1-p_{1}^{2}\right)}{p_{1}^{2}}+\beta \frac{1-p_{1}}{p_{1}}-\lambda_{\max } \frac{1-p_{1}}{p_{1}}\right] z
$$

therefore again the contract has the following form

$$
\begin{aligned}
z & =\frac{c}{\frac{\alpha\left(1-p_{1}^{2}\right)}{p_{1}^{2}}+\beta \frac{1-p_{1}}{p_{1}}-\lambda_{\max } \frac{1-p_{1}}{p_{1}}} \\
y & =\frac{z}{p_{1}} \\
x & =\frac{z}{p_{1}^{2}}
\end{aligned}
$$


Finally to check the upper bound $\frac{p_{1} c}{\left(1-p_{1}\right) \lambda_{\min }(1-\rho)}$ for $z$, one needs to verify

$$
\frac{\alpha\left(1-p_{1}^{2}\right)}{p_{1}}+\beta\left(1-p_{1}\right)-\lambda_{\max }\left(1-p_{1}\right) \geq \lambda_{\min }\left(1-p_{1}\right)(1-\rho)
$$

Canceling $1-p_{1}$, we get

$$
\frac{\alpha\left(1+p_{1}\right)}{p_{1}}+\beta-\lambda_{\max } \geq \lambda_{\min }(1-\rho)
$$

LHS equals to

$$
\lambda_{\min }\left(\rho\left(\frac{1}{p_{1}}-1\right)+1\right) \geq \lambda_{\min }(1-\rho)
$$

which is obvious.

The only part from Proposition 3, which needs proof is that when $\rho \geq \frac{\lambda_{\max }-\lambda_{\min }}{\lambda_{\min }\left(\frac{1}{p_{1}}-1\right)}$, then LP makes more profit by whole-portfolio contracting. This is because, as we saw in the proof above, for these values of $\rho$, the expected profit by GP with whole-portfolio contract is $z+2 c$ for some $z \leq \frac{p_{1} c}{\lambda_{\max }\left(1-p_{1}\right)}$ which is less than $\Pi_{G P}=2 c+\sum_{i=1}^{2} \frac{p_{i} c}{\lambda_{i}\left(1-p_{i}\right)}$ that GP makes under the deal-by-deal contract.

\subsection{Proof of Proposition 5}

As we saw in the Proof of Proposition 2, when $\rho>\rho^{*}$, the expected payout to GP is $z+2 c$. The expression for $z$ in this region is

$$
z=\frac{2 c}{\beta \frac{1-p_{1}}{p_{1}}+\alpha \frac{1-p_{1}^{2}}{p_{1}^{2}}}
$$

So it is only needed to look at how denominator changes when parameters change. Denominator is equal to

$$
\begin{aligned}
& \beta \frac{1-p_{1}}{p_{1}}+\alpha \frac{1-p_{1}^{2}}{p_{1}^{2}} \\
& =\frac{1-p_{1}}{p_{1}}\left[\alpha \frac{1+p_{1}}{p_{1}}+\beta\right]
\end{aligned}
$$

Derivative with respect to $p_{1}$ becomes

$$
-\frac{1}{p_{1}^{2}}\left[\alpha \frac{1+p_{1}}{p_{1}}+\beta\right]+\frac{1-p_{1}}{p_{1}} \times \alpha \frac{-1}{p_{1}^{2}}<0
$$


With respect to $\lambda_{\min }$ and $\lambda_{\max }$, derivatives are $\frac{1-p_{1}}{p_{1}}\left[\rho \frac{1+p_{1}}{p_{1}}+1-2 \rho\right]$ and $\frac{1-p_{1}}{p_{1}}$ and both are positive. This proves the proposition in the region $\rho>\rho^{*}$. In the region $\rho<\rho^{*}$, the expected payout to GP is $\lambda_{\max } y+\left(1-\lambda_{\max }\right) z=\frac{\lambda_{\max }\left(1-p_{1}\right)+p_{1}}{p_{1}} z$. The expression for $z$ in this area is

$$
z=\frac{c}{\frac{\alpha\left(1-p_{1}^{2}\right)}{p_{1}^{2}}+\beta \frac{1-p_{1}}{p_{1}}-\lambda_{\max } \frac{1-p_{1}}{p_{1}}}
$$

with respect to $\lambda_{m i n}$, the derivative of the denominator is same as above. With respect to $\lambda_{\max }$, the derivative of $z$ is zero. However since the expected payout in this regime is $\frac{\lambda_{\max }\left(1-p_{1}\right)+p_{1}}{p_{1}} z$,it is increasing. Finally with respect to $p_{1}$, the denominator for $\frac{1-p_{1}}{p_{1}} z$, is $\frac{\alpha\left(1+p_{1}\right)}{p_{1}}+\beta-\lambda_{\max }$. Derivative with respect to $p_{1}$ of this term is $-\frac{\alpha}{p_{1}^{2}}<0$ hence denominator is decreasing and the whole term is increasing. This finishes the argument.

\subsection{Proof of Proposition 6}

As we mentioned in the discussion proceeding the Proposition 6, in the optimal contract we have

$$
\alpha x+\tilde{\beta} y=\max \left\{\theta_{\max } y+c, p_{1} y+2 c\right\}
$$

By this, LP problem can be written as

$$
\begin{aligned}
& \min _{x, y} \alpha x+\tilde{\beta} y \\
& x \geq y \geq p_{1} x ; \quad \alpha x+\tilde{\beta} y=\max \left\{\theta_{\max } y+c, p_{1} y+2 c\right\}
\end{aligned}
$$

Similar to the proof of Proposition2, we consider two possible cases for $y$.

- If $y \leq \frac{c}{\theta_{\max }-p_{1}}=\frac{c}{\lambda_{\max }\left(1-p_{1}\right)}$, then we $p_{1} y+2 c=\max \left\{\theta_{\max } y+c, p_{1} y+2 c\right\}$, hence one gets

$$
x=f(y)=\frac{\left(p_{1}-\tilde{\beta}\right) y+2 c}{\alpha}
$$

by $p_{1} y+2 c=\alpha x+\tilde{\beta} y$. Therefore LP problem becomes

$$
\begin{aligned}
& \min y \\
& \min \left\{f(y), \frac{c}{\theta_{m}-p_{1}}\right\} \geq y \geq p_{1} f(y)
\end{aligned}
$$

$y \geq p_{1} f(y)$ implies

$$
y \geq \frac{2 p_{1} c}{\alpha-p_{1}\left(p_{1}-\tilde{\beta}\right)}
$$


$x \geq y$ implies

$$
\left(\alpha-\left(p_{1}-\tilde{\beta}\right)\right) y \leq 2 c
$$

if $\alpha-(p-\tilde{\beta})>0$, then

$$
y \leq \frac{2 c}{\left(\alpha-\left(p_{1}-\tilde{\beta}\right)\right)}
$$

otherwise always (25) is satisfied. Note that if $\alpha-(p-\tilde{\beta})>0$, then

$$
\frac{2 p_{1} c}{\alpha-p_{1}\left(p_{1}-\tilde{\beta}\right)} \leq \frac{2 c}{\left(\alpha-\left(p_{1}-\tilde{\beta}\right)\right)}
$$

So there is answer satisfying $y \leq \frac{c}{\theta_{\max }-p_{1}}$ if

$$
\frac{2 p_{1} c}{\alpha-p_{1}\left(p_{1}-\tilde{\beta}\right)} \leq \frac{c}{\theta_{\max }-p_{1}}
$$

In which case $y=\frac{2 p_{1} c}{\alpha-p_{1}\left(p_{1}-\tilde{\beta}\right)}$ and $x=f(y)=\frac{y}{p_{1}}$. Equation (26) holds if

$$
\alpha-p_{1}^{2}+p_{1} \tilde{\beta} \geq 2 \lambda_{\max } p_{1}\left(1-p_{1}\right)
$$

which, after canceling and factoring $\left(1-p_{1}\right)$, becomes

$$
\lambda_{\min }\left[\left(1-p_{1}\right) \rho+p_{1}\right] \geq \lambda_{\max } p_{1}
$$

which is equivalent to

$$
\rho \geq \frac{\lambda_{\max }-\lambda_{\min }}{\lambda_{\min }\left(\frac{1}{p_{1}}-1\right)}
$$

- If $\rho<\frac{\lambda_{\max }-\lambda_{\min }}{\lambda_{\min }\left(\frac{1}{p_{1}}-1\right)}$, then the contract with $y \leq \frac{c}{\lambda_{\max }\left(1-p_{1}\right)}$ is not possible. In this case,

$$
f(y)=x=\frac{\left(\theta_{\max }-\tilde{\beta}\right) y+c}{\alpha}
$$

So LP should solve

$$
\begin{aligned}
& \min y \\
& f(y) \geq y \geq \max \left\{p_{1} f(y), \frac{c}{\lambda_{\max }\left(1-p_{1}\right)}\right\}
\end{aligned}
$$

$y \geq p_{1} f(y)$ implies

$$
y \geq \frac{p_{1} c}{\alpha-p_{1}\left(\theta_{\max }-\tilde{\beta}\right)}
$$


$x \geq y$ implies

$$
\left(\alpha-\left(\theta_{\max }-\tilde{\beta}\right)\right) y \leq c
$$

if $\alpha-\left(\theta_{\max }-\tilde{\beta}\right)>0$ this means

$$
y \leq \frac{c}{(\alpha-(\theta-\tilde{\beta}))}
$$

Otherwise always it is satisfied. In any case, in this region, the possible minimum for $y$ is $\frac{p_{1} c}{\alpha-p_{1}\left(\theta_{\max }-\tilde{\beta}\right)}$, in which case $x=\frac{y}{p_{1}}$.

Finally to show the equality $s^{F N O}(R+I)=s(R+I)$, note that $\tilde{\beta}=\beta+p_{1}(1-\alpha-\beta)$. Hence in the region $\rho<\frac{\lambda_{\max }-\lambda_{\min }}{\lambda_{\min }\left(\frac{1}{p_{1}}-1\right)}$, it is enough to show

$$
\begin{aligned}
& \alpha\left(1-p_{1}^{2}\right)+\beta p_{1}\left(1-p_{1}\right)-\lambda_{\max } p_{1}\left(1-p_{1}\right)= \\
& \alpha-p_{1}\left(\lambda_{\max }+p_{1}\left(1-\lambda_{\max }\right)\right)+p_{1}\left(\beta+p_{1}(1-\alpha-\beta)\right)
\end{aligned}
$$

which is correct after simplification. In the region $\rho \geq \frac{\lambda_{\max }-\lambda_{\min }}{\lambda_{\min }\left(\frac{1}{p_{1}}-1\right)}$ similar algebra works.

\subsection{Proof of Proposition 7}

We use the fact that $s^{F N O}(R+I)=s(R+I)$ which was proved in Proposition 6. I claim, expected payout to both types of GPs are the same under whole-portfolio contracting. For $\rho<\rho^{*}$ both are equal to $p_{1} y+2 c=z+2 c$. In the region $\rho>\rho^{*}$, for non-reputable GP, expected payout is equal to

$$
\begin{aligned}
& \theta_{\max } y+c= \\
& \lambda_{\max } y+\left(1-\lambda_{\max }\right) p_{1} y+c
\end{aligned}
$$

By Proposition 2 is the same as $\lambda_{\max } y+\left(1-\lambda_{\max }\right) z+c$ which is the expected payout to reputable GP by the same proposition. Also as we saw by equation (16) and discussion after it, the expected payouts to both types of GP under deal-by-deal are the same as well. Now in the reputable case, both types of contracting induce the optimal investment strategy of exerting effort and invest only in good project. However for non-reputable GP, wholeportfolio contracting improves total payout of the projects compared to deal-by-deal as we saw in Subsection 4.1. Therefore it can only increase the profit of the LP compared to the reputable case since the expected payout to GP is the same for both types of GPs. This completes the argument. 


\subsection{Proof of Proposition 8}

With given cost functions, in the deal-by-deal, the effort $\lambda_{1}$ is determined by

$$
R-I=a m \lambda_{1}^{m-1}+a m(m-1) \lambda_{1}^{m-1}=a m^{2} \lambda_{1}^{m-1}
$$

so $\lambda_{1}=\sqrt[m-1]{\frac{R-I}{a m^{2}}}$ and the profit from first project is $\lambda_{1}^{2} c^{\prime \prime}\left(\lambda_{1}\right)=a \lambda_{1}^{2} m(m-1) \lambda_{1}^{m-2}$. For the second project we need only to change $a$ to $b$. In the whole-portfolio case, using equations

$$
\begin{aligned}
& b m \lambda_{2}^{m-1}=c_{2}^{\prime}\left(\lambda_{2}\right)=\lambda_{1} s_{G P}(2 R) \\
& a m \lambda_{1}^{m-1}=c_{1}^{\prime}\left(\lambda_{1}\right)=\lambda_{2} s_{G P}(2 R)
\end{aligned}
$$

we have $a m \lambda_{1}^{m}=b m \lambda_{2}^{m}$ which gives $\lambda_{2}=C \lambda_{1}$ where $C=\sqrt[m]{\frac{a}{b}}$. Then second equation above gives

$$
\frac{a}{C} m \lambda_{1}^{m-2}=s_{G P}(2 R)
$$

Therefore LP problem can be written as $\left(\lambda_{1}=\lambda\right)$

$$
\max _{\lambda} C \lambda^{2}\left[2 R-s_{G P}(2 R)\right]+[\lambda(1-C \lambda)+C \lambda(1-\lambda)](R+I)+(1-\lambda)(1-C \lambda) 2 I-2 I
$$

From above $C \lambda^{2} s_{G P}(2 R)=a m \lambda^{m}$ and the profit is

$$
\begin{aligned}
& 2 C \lambda^{2} R-a m \lambda^{m}+\lambda R-C \lambda^{2} R+\lambda I-C \lambda^{2} I \\
& +C \lambda R-C \lambda^{2} R+C \lambda I-C \lambda^{2} I-2 \lambda I-2 C \lambda I+2 C \lambda^{2} I
\end{aligned}
$$

which simplifies to

$$
(\lambda+C \lambda)(R-I)-a m \lambda^{m}
$$

FOC gives

$$
(1+C)(R-I)=a m^{2} \lambda^{m-1}
$$

SO

$$
\lambda_{1}=\lambda=\sqrt[m-1]{\frac{(1+C)(R-I)}{a m^{2}}}
$$

Total profit by LP in this case can be written as

$$
\lambda(1+C)(R-I)\left[1-\frac{1}{m}\right]
$$


So in order to show that LP makes more money with whole-portfolio compared to deal-bydeal, we have to show

$$
\sqrt[m-1]{\frac{(1+C)}{a}}(1+C)>\sqrt[m-1]{\frac{1}{a}}+\sqrt[m-1]{\frac{1}{b}}
$$

since $b=\frac{a}{C^{m}}$, this simplifies to show

$$
\sqrt[m-1]{(1+C)}(1+C)>1+\sqrt[m-1]{C^{m}}
$$

which is equivalent to (set $C=d^{m-1}$ )

$$
\left(1+d^{m-1}\right)^{m}>\left(1+d^{m}\right)^{m-1}
$$

Since the problem is symmetric with respect to $a$ and $b$ we can assume $a \leq b$ hence $d \leq$ 1 ,which makes the inequality above trivial.

\section{Appendix B : Robustness}

\subsection{Increasing Assumption}

The security defined in Proposition 2 is increasing on the set of possible payouts with positive probability (i.e on equilibrium path). However if the payout of good project is not $R$ for sure or GP makes a wrong decision, it is possible to get a payout of size $R$. To show robustness of our main result on the relation between correlation and security design, we have the following proposition.

Proposition 13 If the condition $s(R) \geq s(2 I)$ is imposed to the security $s_{G P}=s$, the result of Proposition 圆 remains unchanged if $\frac{1}{4} \geq p_{1} \geq p_{2}$.

\section{Proof.}

Take $(z, w, x, y)=(s(2 I), s(R), s(R+I), s(2 R))$. Similar to the case of security with no restriction, in the optimum $s(I)=0$. Also since $R$ is not outcome of optimal investment strategy, it should be the lowest possible value such that the security remains increasing hence $s(R)=s(2 I)=z$. With the same reason as discussed in subsection 3.2, to motivate 
for optimal investment strategy, contract should satisfy

$$
\begin{aligned}
& z \geq p_{1} p_{2} x+\left[p_{1}\left(1-p_{2}\right)+p_{2}\left(1-p_{1}\right)\right] z, p_{1} y \\
& y \geq p_{1} x+\left(1-p_{1}\right) z \\
& x \geq z, y
\end{aligned}
$$

Also to motivate effort,

$$
\begin{aligned}
& \rho \lambda_{\min } x+\left[\lambda_{1}-2 \rho \lambda_{\min }+\lambda_{2}\right] y+\left[1-\lambda_{1}-\lambda_{2}+\rho \lambda_{\min }\right] z \\
& \geq z+2 c, \lambda_{\max } y+\left(1-\lambda_{\max }\right) z+c
\end{aligned}
$$

Therefore LP problem is

$$
\min _{x, y, z} \alpha x+\beta y+\gamma z
$$

subject to conditions above. Here as before, $(\alpha, \beta, \gamma)=\left(\rho \lambda_{\min }, \lambda_{1}-2 \rho \lambda_{\min }+\lambda_{2}, 1-\lambda_{1}-\lambda_{2}+\rho \lambda_{\min }\right)$. With similar argument as in the main case, we have

$$
\begin{aligned}
& \alpha x+\beta y+\gamma z=\max \left\{z+2 c, \lambda_{\max } y+\left(1-\lambda_{\max }\right) z+c\right\} \\
& z=\max \left\{p_{1} p_{2} x+\left[p_{1}\left(1-p_{2}\right)+p_{2}\left(1-p_{1}\right)\right] z, p_{1} y\right\}
\end{aligned}
$$

Based on which terms becomes maximum on the RHS of the first equality, I consider the following two cases

1. First consider the case $z \leq \frac{p_{1} c}{\lambda_{\max }\left(1-p_{1}\right)}$. In this case the maximum payout will be $z+2 c$ so we have

$$
\alpha x+\beta y+\gamma z=z+2 c
$$

by this

$$
x=\frac{z(1-\gamma)-\beta y+2 c}{\alpha}
$$

Now divide this case to two sub-cases.

- $p_{1} y=\max \left\{p_{1} p_{2} x+\left[p_{1}\left(1-p_{2}\right)+p_{2}\left(1-p_{1}\right)\right] z, p_{1} y\right\}=z$. As the result

$$
x=\frac{z\left(1-\gamma-\frac{\beta}{p_{1}}\right)+2 c}{\alpha}
$$


hence the problem for LP can be written as

$$
\begin{aligned}
& \min z \\
& x \geq y=\frac{z}{p_{1}} \\
& y \geq p_{1} x+\left(1-p_{1}\right) z \\
& z=p_{1} y \geq p_{1} p_{2} x+\left[p_{1}\left(1-p_{2}\right)+p_{2}\left(1-p_{1}\right)\right] z
\end{aligned}
$$

The last two inequalities can be written as

$$
\begin{aligned}
& z \geq \frac{p_{1}^{2}}{1-p_{1}+p_{1}^{2}} x=\theta_{1} x \\
& z \geq \frac{p_{1} p_{2}}{1-p_{1}+2 p_{1} p_{2}-p_{2}} x=\theta_{2} x
\end{aligned}
$$

RHS of the second inequality is increasing in $p_{2}$. So if $p_{2} \leq p^{*}$, for some $p^{*} \geq 0$, the first inequality is effective otherwise the second one is. In any case, we get

$$
\alpha z / \theta_{i} \geq z\left(1-\gamma-\frac{\beta}{p_{1}}\right)+2 c
$$

so for optimal $z$ we have

$$
z=\frac{2 c}{\beta \frac{1-p_{1}}{p_{1}}+\alpha \frac{1-\theta_{\max }}{\theta_{\max }}}
$$

which is similar to the formula as in Proposition 2, When comparing with the initial inequality, we get

$$
\alpha \frac{1-\theta_{i}}{\theta_{i}}+\beta \frac{1-p_{1}}{p_{1}} \geq \frac{2 \lambda_{\max }\left(1-p_{1}\right)}{p_{1}}
$$

so similar to the main case, if we have

$$
\alpha \frac{1-\theta_{i}}{\theta_{i}}+\beta \frac{1-p_{1}}{p_{1}}
$$

is increasing in $\rho$, then we have shown the proposition. The derivative with respect to $\rho$ is

$$
\lambda_{\text {min }}\left(\frac{1-\theta_{i}}{\theta_{i}}-2 \frac{1-p_{1}}{p_{1}}\right)
$$

Maximum value for $\theta_{i}$ is when $p_{1}=p_{2}$ and for this case by $p_{1} \leq \frac{1}{4}$ we get $\frac{1-\theta_{i}}{\theta_{i}} \geq 2 \frac{1-p_{1}}{p_{1}}$ hence the derivative above is always positive. This shows that payout to LP is increasing in $\rho$ in this case. 
- $p_{1} p_{2} x+\left[p_{1}\left(1-p_{2}\right)+p_{2}\left(1-p_{1}\right)\right] z=\max \left\{p_{1} p_{2} x+\left[p_{1}\left(1-p_{2}\right)+p_{2}\left(1-p_{1}\right)\right] z, p_{1} y\right\}=z$.

From this we get

$$
x=\frac{1-\left[p_{1}\left(1-p_{2}\right)+p_{2}\left(1-p_{1}\right)\right]}{p_{1} p_{2}} z=q z
$$

on the other hand, we have

$$
x=\frac{z(1-\gamma)-\beta y+2 c}{\alpha}
$$

so we have

$$
\beta y=2 c-\alpha q z+z(1-\gamma)
$$

which gives $y$ in terms of $z$. So LP problem is

$\min z$

where $p_{1} y \leq z$ and $y \geq p_{1} x+\left(1-p_{1}\right) z=\left(p_{1} q+1-p_{1}\right) z$. If the coefficient in the later inequality is bigger than $\frac{1}{p_{1}}$, there is no possible solution. Otherwise, when insert $y$ from equality above in the inequality $p_{1} y \leq z$, in the optimal it binds hence $p_{1} y=z$ and problem reduces to the previous case.

2. Now consider the case $z \geq \frac{p_{1} c}{\lambda_{\max }\left(1-p_{1}\right)}$. As saw above this happens for small $\rho$. In this case we have

$$
\begin{aligned}
& \alpha x+\beta y+\gamma z=\max \left\{z+2 c, \lambda_{\max } y+\left(1-\lambda_{\max }\right) z+c\right\} \\
& =\lambda_{\max } y+\left(1-\lambda_{\max }\right) z+c
\end{aligned}
$$

like in the previous case, we consider two sub-cases.

- $p_{1} y=\max \left\{p_{1} p_{2} x+\left[p_{1}\left(1-p_{2}\right)+p_{2}\left(1-p_{1}\right)\right] z, p_{1} y\right\}=z$. Then the equation for expected payout can be written as

$$
x=\frac{\left(\frac{\lambda_{\max }}{p_{1}}+1-\lambda_{\max }-\frac{\beta}{p_{1}}-\gamma\right) z+c}{\alpha}
$$


similar to the previous case, LP problem becomes

$$
\begin{aligned}
& \min z \\
& x \geq y=\frac{z}{p_{1}} \\
& y \geq p_{1} x+\left(1-p_{1}\right) z \\
& z=p_{1} y \geq p_{1} p_{2} x+\left[p_{1}\left(1-p_{2}\right)+p_{2}\left(1-p_{1}\right)\right] z
\end{aligned}
$$

$p_{1} x \geq z$ implies that

$$
p_{1} c \geq\left(\alpha+\beta-\lambda_{\max }\right)\left(1-p_{1}\right) z
$$

which in turns implies that

$$
z \leq \frac{p_{1} c}{\left(1-p_{1}\right) \lambda_{\min }(1-\rho)}
$$

similar to the previous case, we can write the last two inequalities as

$$
\begin{aligned}
& z \geq \frac{p_{1}^{2}}{1-p_{1}+p_{1}^{2}} x=\theta_{1} x \\
& z \geq \frac{p_{1} p_{2}}{1-p_{1}+2 p_{1} p_{2}-p_{2}} x=\theta_{2} x
\end{aligned}
$$

In any case this gives us

$$
\alpha z / \theta_{i} \geq\left(\frac{\lambda_{\max }}{p_{1}}+1-\lambda_{\max }-\frac{\beta}{p_{1}}-\gamma\right) z+c
$$

which gives us the optimal $z$ as the bigger term of two inequalities above is binding. Hence we have

$$
z=\frac{c}{\frac{\alpha\left(1-\theta_{\max }\right)}{\theta_{\max }}+\beta \frac{1-p_{1}}{p_{1}}-\lambda_{\max } \frac{1-p_{1}}{p_{1}}}
$$

Again this is similar to the formula we have as in Proposition 2 and with the same reason as above $z$ is decreasing in $\rho$ which shows our claim in this case.

- $p_{1} p_{2} x+\left[p_{1}\left(1-p_{2}\right)+p_{2}\left(1-p_{1}\right)\right] z=\max \left\{p_{1} p_{2} x+\left[p_{1}\left(1-p_{2}\right)+p_{2}\left(1-p_{1}\right)\right] z, p_{1} y\right\}=z$. As previous case, this gives

$$
x=\frac{1-\left[p_{1}\left(1-p_{2}\right)+p_{2}\left(1-p_{1}\right)\right]}{p_{1} p_{2}} z=q z
$$


On the other hand we have

$$
\alpha x+\beta y+\gamma z=\lambda_{\max } y+\left(1-\lambda_{\max }\right) z+c
$$

which gives

$$
\left(\beta-\lambda_{\max }\right) y=\left(1-\lambda_{\max }-\gamma-\alpha q\right) z+c
$$

so LP problem is

$\min z$

$p_{1} y \leq z$ and $y \geq p_{1} x+\left(1-p_{1}\right) z=\left(p_{1} q+1-p_{1}\right) z$. Similar to the case we studied before either this does not have solution or we get $p_{1} y=z$ in the optimum hence it reduces to the previous case. This finishes the argument.

\subsection{Return Distribution}

Here I want to generalize the distribution function for the projects. Let's assume the support of both types of projects $G$ and $B$ are $0, R_{1}, R_{2}$. For the $G$ type the chances are $\{0, p, 1-p\}$ respectively and for the $B$ type it is $\left\{1-p_{1}-p_{2}, p_{1}, p_{2}\right\}$. All the other variables, definitions and assumptions are the same as in the main model. First want to see how contract on one project is written. In order to persuade the optimal investment strategy (not investing on bad project), we should have

$$
s_{G P}(I) \geq p_{1} s_{G P}\left(R_{1}\right)+p_{2} s_{G P}\left(R_{2}\right)
$$

Also in order to motivate effort, we have

$$
E\left[s_{G P}(G)\right]=p s_{G P}\left(R_{1}\right)+(1-p) s_{G P}\left(R_{2}\right) \geq s_{G P}(I)+\frac{c}{\lambda}
$$

In the optimal, with the same reasoning as in the binary case, both these inequalities are binding to minimize the expected payout to GP. LP problem is

$$
\min _{s_{G P}\left(R_{1}\right), s_{G P}\left(R_{2}\right)} \lambda E\left[s_{G P}(G)\right]+(1-\lambda) s_{G P}(I)
$$


Conditioned to equations (equities in optimum) (27) and (28). Because of optimality, LP problem can be written as

$$
\min _{x, y}\left[\lambda p+(1-\lambda) p_{1}\right] x+\left[\lambda(1-p)+(1-\lambda) p_{2}\right] y
$$

where $(x, y)=\left(s_{G P}\left(R_{1}\right), s_{G P}\left(R_{2}\right)\right)$. The relation between $x$ and $y$ comes from (28) above which can be written as $x=\gamma y+\zeta$ where

$$
\begin{aligned}
& \gamma=-\frac{1-p-p_{2}}{p-p_{1}} \\
& \zeta=\frac{c}{\lambda}
\end{aligned}
$$

So the minimization problem is linear in $y$ and hence in the optimum either we have $x=0$ or $y=0$ as none of payouts can be negative. More precisely, the coefficient of $y$ in the LP problem is

$$
-\left[\lambda p+(1-\lambda) p_{1}\right] \frac{1-p-p_{2}}{p-p_{1}}+\left[\lambda(1-p)+(1-\lambda) p_{2}\right]
$$

if this coefficient is positive then we should have $y=0$ otherwise $x=0$. Whichever happens, we get the value of the other variable from equation $x=\gamma y+\zeta$ above. If the payout of the projects has $n$ different values, the same conclusion holds as the problem is linear in payouts. In summary we have

Proposition 14 With setup as above, the optimal contract satisfies either $s\left(R_{1}\right)=0$ or $s\left(R_{2}\right)=0$. In particular $s\left(R_{2}\right)=0$ if and only if

$$
\left[\lambda(1-p)+(1-\lambda) p_{2}\right]\left(p-p_{1}\right)>\left[\lambda p+(1-\lambda) p_{1}\right]\left(1-p-p_{2}\right)
$$

The other one is computed by the equation $x=\gamma y+\zeta$, where $\gamma, \zeta$ are given in equations (29). Finally $s(I)$ is computed from the equation (27) when it is equality.

Let's just explain the intuition behind the property that either $s\left(R_{1}\right)=0$ or $s\left(R_{2}\right)=0$. LP wants to minimize the incentive for the GP to invest in a bad project. To do this, LP considers the (weighted) difference of probabilities that either the outcome is $R_{1}$ or $R_{2}$. Then he makes no payment in the state with lower chance. The other outcome associates to higher chance of investing in the good project so GP motivates it in the contract.

Now I look at the whole-portfolio problem. Again we assume that the policy implemented is optimal so we have $s(I)=s\left(R_{1}\right)=s\left(R_{2}\right)=0$. Possible returns from optimal strategy are $2 I$ when two projects are bad, $R_{i}+I$ when one is good and other is bad and finally $2 R_{i}$ or $R_{1}+R_{2}$ 
when both are good. I assume parameters for the first projects are $\lambda_{1}, p, p_{1}$ and $p_{2}$. For the second one $\lambda_{2}, q, q_{1}$ and $q_{2}$. I show the payouts to GP by $z=s(2 I), y_{i}=s\left(R_{i}+I\right), x_{i}=s\left(2 R_{i}\right)$ and $x=s\left(R_{1}+R_{2}\right)$. I assume correlation $\rho$ between good projects as in the main case and assume that for good projects the realization of the returns are independent. We have

$$
\begin{aligned}
& s(2 I) \geq \sum p_{i} s\left(R_{i}+I\right), \sum q_{i} s\left(R_{i}+I\right), \sum p_{i} q_{j} s\left(R_{i}+R_{j}\right) \\
& p s\left(R_{1}+I\right)+(1-p) s\left(R_{2}+I\right)=E_{G_{1}}\left[s\left(R_{i}+I\right)\right] \geq \\
& s(2 I),\left[p q_{2}+(1-p) q_{1}\right] s\left(R_{1}+R_{2}\right)+p q_{1} s\left(2 R_{1}\right)+(1-p) q_{2} s\left(2 R_{2}\right) \\
& q s\left(R_{1}+I\right)+(1-q) s\left(R_{2}+I\right)=E_{G_{2}}\left[s\left(R_{i}+I\right)\right] \geq \\
& s(2 I),\left[q p_{2}+(1-q) p_{1}\right] s\left(R_{1}+R_{2}\right)+q p_{1} s\left(2 R_{1}\right)+(1-q) p_{2} s\left(2 R_{2}\right) \\
& {[p(1-q)+q(1-p)] s\left(R_{1}+R_{2}\right)+p q s\left(2 R_{1}\right)+(1-p)(1-q) s\left(2 R_{2}\right)} \\
& \geq E_{G_{j}}\left[s\left(R_{i}+I\right)\right]
\end{aligned}
$$

And finally LP should impose the equation which motivates effort. This can be written as

$$
\begin{aligned}
& \rho \lambda_{\min } E_{G_{1}, G_{2}}\left[s\left(R_{i}+R_{j}\right)\right]+\left(\lambda_{\max }-\rho \lambda_{\text {min }}\right) E_{G_{\max }}\left[s\left(R_{i}+I\right)\right] \\
& +(1-\rho) \lambda_{\min } E_{G_{\min }}\left[s\left(R_{i}+I\right)\right]+\left(1-\lambda_{1}-\lambda_{2}+\rho \lambda_{\min }\right) s(2 I) \\
& \geq z+2 I, \lambda_{\max } E_{G_{\max }}\left[s\left(R_{i}+I\right)\right]+\left(1-\lambda_{\max }\right) z+c, \lambda_{\min } E_{G_{\min }}\left[s\left(R_{i}+I\right)\right]+\left(1-\lambda_{\min }\right) z+c
\end{aligned}
$$

As in the binary case, LP wants to minimize the expected payout to GP (LHS of the last inequality) given constraints above. Similar to the binary case, we can see that $E_{G_{1}, G_{2}}\left[s\left(R_{i}+\right.\right.$ $\left.R_{j}\right)$ ] can be represented with an inequality. So LP problem can be written as

$$
\min E_{G_{1}, G_{2}}\left[s\left(R_{i}+R_{j}\right)\right]
$$

subject to conditions for optimal investment and motivation for effort. Since the general problem seems hard to solve and get good intuition from, I stick to two important especial cases.

1. Suppose either $p_{1}$ and $q_{1}$ are small or $p_{2}$ and $q_{2}$ are small. In this case, suppose LP changes compensations for GP in the case of two successful investment, while expected payout remains the same. By this I mean changing $x_{i}$ and $x$ such that

$$
[p(1-q)+q(1-p)] x+p q x_{1}+(1-p)(1-q) x_{2}
$$

remains fixed. By this change, RHS of the equations for optimal investment in case of one success or no success can be changed (The first three equation in the set of 
equations (30) ) . As long as RHS becomes smaller in these equations, the change can be good (or have no effect if conditions are not binding on them). So it is better for GP to consider payouts to minimize RHS of motivating equations. So in this case, in the optimal contract, we get only $x_{i}>0$ (and $x_{j}, x$ are zero) when $p_{i}$ and $q_{i}$ are small. Similar reasoning implies only $y_{i}>0$ and $y_{j}=0$ when $p_{i}$ and $q_{i}$ are small. Hence in this case problem reduces effectively to the binary case.

2. Now consider the orthogonal problem to what we discussed in the previous part. So I assume $p_{2}=q_{1}=0$ so the first bad project only have return $R_{1}$ and the second one only $R_{2}$. Equations (30) are reduced to

$$
\begin{aligned}
& s(2 I) \geq p_{1} s\left(R_{1}+I\right), q_{2} s\left(R_{2}+I\right), p_{1} q_{2} s\left(R_{1}+R_{2}\right) \\
& p s\left(R_{1}+I\right)+(1-p) s\left(R_{2}+I\right)=E_{G_{1}}\left[s\left(R_{i}+I\right)\right] \geq \\
& s(2 I), p q_{2} s\left(R_{1}+R_{2}\right)+(1-p) q_{2} s\left(2 R_{2}\right) \\
& q s\left(R_{1}+I\right)+(1-q) s\left(R_{2}+I\right)=E_{G_{2}}\left[s\left(R_{i}+I\right)\right] \geq \\
& s(2 I),(1-q) p_{1} s\left(R_{1}+R_{2}\right)+q p_{1} s\left(2 R_{1}\right) \\
& {[p(1-q)+q(1-p)] s\left(R_{1}+R_{2}\right)+p q s\left(2 R_{1}\right)+(1-p)(1-q) s\left(2 R_{2}\right)} \\
& \geq E_{G_{j}}\left[s\left(R_{i}+I\right)\right]
\end{aligned}
$$

In this case if we get $s(2 I)=z$, then similar reasoning as in binary case,

$$
\begin{aligned}
& z=p_{1} s\left(R_{1}+I\right)=p_{1}^{2} s\left(2 R_{1}\right) \\
& z=q_{2} s\left(R_{2}+I\right)=q_{2}^{2} s\left(2 R_{2}\right) \\
& z=p_{1} q_{2} s\left(R_{1}+R_{2}\right)
\end{aligned}
$$

which implies that, as in the main case, $E\left[s_{G P}\right]$ is decreasing in $\rho$ as well. 